\title{
Faddeev and Glauber Calculations at Intermediate Energies in a Model for $\mathbf{n}+\mathbf{d}$ Scattering
}

\author{
Ch. Elster ${ }^{(a)}$, T. Lin ${ }^{(a)}$, W. Glöckle ${ }^{(b)}$, and S. Jeschonnek ${ }^{(c)}$ \\ (a) Institute of Nuclear and Particle Physics, and Department of \\ Physics and Astronomy, Ohio University, Athens, OH 45701, USA \\ (b) Institute for Theoretical Physics II, Ruhr-University Bochum, D-44780 Bochum, Germany and \\ (c) Department of Physics, The Ohio State University, Lima, OH 45804, USA
}

(Dated: October 27, 2018)

\begin{abstract}
Obtaining cross sections for nuclear reactions at intermediate energies based on the Glauber formulation has a long tradition. Only recently the energy regime of a few hundred $\mathrm{MeV}$ has become accessible to ab-initio Faddeev calculations of three-body scattering. In order to go to higher energies, the Faddeev equation for three-body scattering is formulated and directly solved without employing a partial wave decomposition. In the simplest form the Faddeev equation for interacting scalar particles is a three-dimensional integral equation in five variables, from which the total cross section, the cross sections for elastic scattering and breakup reactions, as well as differential cross sections are obtained. The same observables are calculated based on the Glauber formulation. The first order Glauber calculation and the Glauber rescattering corrections are compared in detail with the corresponding terms of the Faddeev multiple scattering series for projectile energies between $100 \mathrm{MeV}$ and $2 \mathrm{GeV}$.
\end{abstract}

PACS numbers: 21.45-v,24.10.Ht,25.10.+s

\section{INTRODUCTION}

The formulation of high energy scattering introduced by Glauber [1, 2] has a long tradition in being applied to calculating nuclear reactions with hadronic as well as electromagnetic probes. Today Glauber amplitudes are widely used to account for final state interactions in (e,e'p) reactions at high and intermediate energies [3, 4, 5]. Glauber theory is applied to investigate color transparency, in $\left(e, e^{\prime} p\right)$ reactions and in the electroproduction of mesons [6, 7]. In addition, it is applied to heavy ion reactions [8]. Most recently, several generalizations of Glauber theory have been employed for the description of electron scattering mainly from lighter nuclei, e.g. the generalized eikonal approximation (GEA) 9, 10], or relativistic eikonal approaches [11, 12]. These methods are applied both to $A\left(e, e^{\prime} p\right)$ and $A(p, 2 p)$ reactions. Nuclear scattering at intermediate energies in the context of radioactive beams also takes advantage of this formulation [13, 14].

Despite the widespread use of the Glauber formulation in reactions involving few as well as many-body systems there has been little work on rigorous tests of the accuracy and/or limits of the Glauber ansatz, apart from some studies concerning the quality of the eikonal approximation for $\left(e, e^{\prime} p\right)$ reactions [15] as well as for elastic proton scattering from halo nuclei [16].

Only recently exact Faddeev calculations for three-body scattering in the intermediate energy regime became available. This progress is based on a formulation of the Faddeev equations, which is based directly on momentum variables and does not rely on traditional partial wave expansions. The formulation and numerical realization for the nonrelativistic Faddeev equations [17, 18] as well as fully Poincaré invariant ones [19, 20, 21] have been carried out for scalar interactions up to projectile energies of $2 \mathrm{GeV}$. This now allows us to perform a detailed comparison of three-body Faddeev calculations of total and elastic cross sections with the ones obtained by Glauber calculations, and thus test the range of validity of Glauber calculations at least in the three-body system.

The naive expectation is that for sufficiently high energies the total $n+d$ cross section is just the sum of the two total cross sections for two-nucleon scattering. The question whether this is valid is an old one and has been investigated in the context of the eikonal approximation [1, 25, 26, 27]. It turns out that rescattering (shadowing) corrections need to be added. Since at the time no exact calculations were available, the question remained open, whether those corrections were sufficient and at which energy the eikonal approximation became valid. We will investigate such questions in this work and mostly follow the formulation given in Refs. [25, 26]. In order to make closer contact with that work as well as for transparency, we will use the nonrelativistic formulation of the Faddeev equation for our comparison, even though we carry out calculations in the intermediate energy regime. We will also use the same two-body scattering amplitude as well as deuteron wave function in the Glauber calculation as enters in our Faddeev results, so that we can clearly identify the effects of the Glauber approximation on the multiple scattering. In Section II we will briefly present the Faddeev framework and its multiple scattering expansion, and in Section III we will re-derive the essential expressions of the Glauber approximation necessary for the comparison to the Faddeev formulation. Our numerical results for the total and differential cross section for elastic scattering in both formulations 
and their discussion will be given in Section IV. We summarize and conclude in Section V.

\section{THE FADDEEV MULTIPLE SCATTERING SERIES}

Various presentations of three-body scattering in the Faddeev scheme are presented in the literature [22, 23]. We solve the Faddeev equation for three identical particles in the form

$$
T|\phi\rangle=t P|\phi\rangle+t P G_{0} T|\phi\rangle
$$

The driving term of this integral equation consists of the two-body t-matrix $t$, the sum $P=P_{12} P_{23}+P_{13} P_{23}$ of a cyclic and anti-cyclic permutation of three particles, and the initial state $|\phi\rangle=\left|\phi_{d} \mathbf{q}_{0}\right\rangle$, composed of a two-body bound state $\phi_{d}$ and the momentum eigenstate of the projectile particle. The kernel of Eq. (2.1) contains the free three-body propagator, $G_{0}=\left(E-H_{0}+i \varepsilon\right)^{-1}$, where $E$ is the total energy in the center-of-momentum (c.m.) frame.

The operator $T$ determines both, the full break-up amplitude

$$
U_{0}=(1+P) T
$$

and the amplitude for elastic scattering

$$
U=P G_{0}^{-1}+P T
$$

In this paper we focus on three identical bosons and use a momentum space representation. For solving Eq. (2.1), we introduce the standard Jacobi momenta $\mathbf{p}$ for the relative momentum in the subsystem, and $\mathbf{q}$ for the relative momentum of the spectator to the subsystem. The momentum states are normalized according to $\left\langle\mathbf{p}^{\prime} \mathbf{q}^{\prime} \mid \mathbf{p q}\right\rangle=$ $\delta^{3}\left(\mathbf{p}^{\prime}-\mathbf{p}\right) \delta^{3}\left(\mathbf{q}^{\prime}-\mathbf{q}\right)$. Projecting Eq. 2.1) onto Jacobi momenta leads to [17]

$$
\begin{aligned}
\left\langle\mathbf{p q}|T| \phi_{d} \mathbf{q}_{0}\right\rangle & =\phi_{d}\left(\mathbf{q}+\frac{1}{2} \mathbf{q}_{0}\right) t_{s}\left(\mathbf{p}, \frac{1}{2} \mathbf{q}+\mathbf{q}_{0}, E-\frac{3}{4 m} q^{2}\right) \\
& +\int d^{3} q^{\prime \prime} t_{s}\left(\mathbf{p}, \frac{1}{2} \mathbf{q}+\mathbf{q}^{\prime \prime}, E-\frac{3}{4 m} q^{2}\right) \frac{\left\langle\mathbf{q}+\frac{1}{2} \mathbf{q}^{\prime \prime}, \mathbf{q}^{\prime \prime}|T| \phi_{d} \mathbf{q}_{0}\right\rangle}{E-\frac{1}{m}\left(q^{2}+q^{\prime \prime 2}+\mathbf{q} \cdot \mathbf{q}^{\prime \prime}\right)+i \varepsilon}
\end{aligned}
$$

Here $t_{s}\left(\mathbf{p}^{\prime}, \mathbf{p}\right)=t\left(\mathbf{p}, \mathbf{p}^{\prime}\right)+t\left(-\mathbf{p}^{\prime}, \mathbf{p}\right)$ is the symmetrized two-body $t$ matrix and the total energy $E$ is explicitly given as

$$
E=E_{d}+\frac{3}{4 m} q_{0}^{2}=E_{d}+\frac{2}{3} E_{l a b} .
$$

We assume that the underlying two-body force generates a $t$-matrix as solution of a two-body Lippmann-Schwinger equation, and that the force supports one bound state with energy $E_{d}$. Then Eq. (2.4) is solved directly in momentum space as function of the vector Jacobi momenta and angles between them. For further details we refer to Ref. [17].

In this work we want to study the high energy behavior of the cross sections. In the framework of the Faddeev formulation, we consider the multiple scattering series defined by the integral equation given in Eq. (2.1). For the elastic scattering amplitude this leads to the series expansion

$$
\langle\Phi|U| \Phi\rangle=\left\langle\Phi\left|P G_{0}^{-1}\right| \Phi\right\rangle+\langle\Phi|P t P| \Phi\rangle+\left\langle\Phi\left|P t P G_{0} t P\right| \Phi\right\rangle+\cdots
$$

which for the fully converged solution is summed as Padé or Neumann series. Via the optical theorem the total cross section is related to the imaginary part of the operator $U$ as

$$
\sigma_{\text {tot }}^{\mathrm{ND}}=-(2 \pi)^{3} \frac{4 m}{3 q_{0}} \Im m\langle\Phi|U| \Phi\rangle .
$$

This again can be expanded in a multiple scattering series through

$$
\begin{aligned}
2 i \Im m\langle\Phi|U| \Phi\rangle & =\langle\Phi|U| \Phi\rangle-\langle\Phi|U| \Phi\rangle^{\star} \\
& =\left\langle\Phi\left|P\left(t-t^{\dagger}\right) P\right| \Phi\right\rangle+\left\langle\Phi\left|P t P G_{0} t P\right| \Phi\right\rangle-\left\langle\Phi\left|P t^{\dagger} G_{0}^{*} P t^{\dagger} P\right| \Phi\right\rangle+\cdots
\end{aligned}
$$

It turns out that the Faddeev multiple scattering series for the total cross sections converges fast for projectile energies higher than $500 \mathrm{MeV}$ [17, 21]. This finding is encouraging for our study of the Glauber formulation. 
One can also start from Eq. (2.8) and derive a high-energy limit in the frame work of the Faddeev equations. This has been carried out in Ref. [24]. Here the terms of first and second order in $t$ have been investigated in the limit $E \rightarrow \infty$ leading to the analytic result

$$
\begin{aligned}
\sigma_{\text {tot }}^{\mathrm{ND}} & =2 \sigma_{\text {tot }}^{\mathrm{NN}} \\
& +(2 \pi)^{5}\left(\frac{4 m}{3 q_{0}}\right)^{2}\left[\Re e\left\langle\frac{3}{4} \mathbf{q}_{\mathbf{0}}\left|t\left(\frac{1}{m}\left(\frac{3}{4} q_{0}\right)^{2}\right)\right| \frac{3}{4} \mathbf{q}_{\mathbf{0}}\right\rangle\right]^{2}\left\langle\phi_{d}\left|\frac{1}{r^{2}}\right| \phi_{d}\right\rangle \frac{1}{4 \pi}-\left(\sigma_{t o t}^{\mathrm{NN}}\right)^{2}\left\langle\phi_{d}\left|\frac{1}{r^{2}}\right| \phi_{d}\right\rangle \frac{1}{4 \pi} \\
& =2 \sigma_{\text {tot }}^{\mathrm{NN}}+O\left(t^{2}\right)+O\left(t^{4}\right)
\end{aligned}
$$

In first order this expansion gives twice the two-nucleon cross section. The second order correction terms can now be tested against exact Faddeev calculations. In Ref. [24] this was not possible.

\section{THE GLAUBER CALCULATION}

For the convenience of the reader we briefly sketch the derivation of the Glauber amplitude for elastic $n+d$ scattering, following the work given in Refs. [25, 26]. In the laboratory system with the projectile momentum $\mathbf{k}$ and the momentum transfer $\mathbf{q}=\mathbf{k}-\mathbf{k}^{\prime}$ the two- particle scattering amplitude according to Glauber [1] is assumed to have the form

$$
f_{k}(\mathbf{q})=\frac{i k}{2 \pi} \int d^{2} b e^{i \mathbf{q} \cdot \mathbf{b}} \Gamma(\mathbf{b})
$$

where $\Gamma(\mathbf{b})$ is given through the eikonal phase $\chi(\mathbf{b})$ as

$$
\Gamma(\mathbf{b})=1-e^{i \chi(\mathbf{b})} .
$$

The vector $\mathbf{b}$ is assumed to be perpendicular to a direction $\hat{n}$, specified below. Scattering off a deuteron target then leads to

$$
F_{k}(\mathbf{q})=\frac{i k}{2 \pi} \int d^{2} b e^{i \mathbf{q} \cdot \mathbf{b}} \int d^{3} r \phi_{d}^{*}(\mathbf{r})\left(1-e^{i(\chi(\mathbf{b}-1 / 2 \mathbf{s})+\chi(\mathbf{b}+1 / 2 \mathbf{s}))}\right) \phi_{d}(\mathbf{r}),
$$

where $\phi_{d}(\mathbf{r})$ is the deuteron wave function and $\mathbf{r}=\mathbf{s}+\hat{n}(\hat{n} \cdot \mathbf{r})$. Using these definitions it follows that

$$
\begin{aligned}
F_{k}(\mathbf{q})= & \frac{i k}{2 \pi} \int d^{2} b e^{i \mathbf{q} \cdot \mathbf{b}} \int d^{3} r \phi_{d}^{*}(\mathbf{r}) \\
& {\left[\Gamma\left(\mathbf{b}-\frac{1}{2} \mathbf{s}\right)+\Gamma\left(\mathbf{b}+\frac{1}{2} \mathbf{s}\right)-\Gamma\left(\mathbf{b}-\frac{1}{2} \mathbf{s}\right) \Gamma\left(\mathbf{b}+\frac{1}{2} \mathbf{s}\right)\right] \phi_{d}(\mathbf{r}) }
\end{aligned}
$$

Using the inverse of Eq. (3.1),

$$
\Gamma(\mathbf{b})=\frac{1}{2 \pi i k} \int d^{2} q e^{-i \mathbf{q} \cdot \mathbf{b}} f_{k}(\mathbf{q})
$$

and again integrating over a vector $\mathbf{q}$ in a plane perpendicular to $\hat{n}$ one can eliminate the $\Gamma$ 's in favor of the two-particle scattering amplitude leading to the expression given in Refs. [25, 26]

$$
F_{k}(\mathbf{q})=2 S\left(\frac{1}{2} \mathbf{q}\right) f(\mathbf{q})+\frac{i}{2 \pi k} \int d^{2} q^{\prime} S\left(\mathbf{q}^{\prime}\right) f_{k}\left(\frac{1}{2} \mathbf{q}+\mathbf{q}^{\prime}\right) f_{k}\left(\frac{1}{2} \mathbf{q}-\mathbf{q}^{\prime}\right) .
$$

The deuteron wave function occurs in the form factor

$$
S(\mathbf{q})=\int d^{3} r\left|\phi_{d}(\mathbf{r})\right|^{2} e^{i \mathbf{q} \cdot \mathbf{s}}=\int d^{3} r\left|\phi_{d}(\mathbf{r})\right|^{2} e^{i \mathbf{q} \cdot \mathbf{r}} .
$$

From the above expression one obtains the laboratory differential cross section for $n+d$ scattering as

$$
\frac{d \sigma^{\mathrm{ND}}}{d \Omega_{l}}=\left|F_{k}(\mathbf{q})\right|^{2}
$$


and the total cross section for $n+d$ scattering

$$
\sigma_{t o t}^{\mathrm{ND}}=\frac{4 \pi}{k} \Im m F_{k}(\mathbf{0})
$$

In first order in the two-body scattering amplitude Eq. (3.9) leads to the expected result for the total cross section

$$
\sigma_{t o t}^{\mathrm{ND}, 1 s t}=2 S(0) \frac{4 \pi}{k} \Im m f_{k}(0)=2 \frac{4 \pi}{k} \Im m f_{k}(0)=2 \sigma_{\text {tot }}^{\mathrm{NN}}
$$

and to the differential cross section for elastic $n+d$ scattering

$$
\frac{d \sigma^{\mathrm{ND}, 1 s t}}{d \Omega_{l}}=4 S^{2}\left(\frac{1}{2} \mathbf{q}\right)\left|f_{k}(\mathbf{q})\right|^{2}
$$

For the total cross section for $n+d$ scattering, including the second order correction, one obtains

$$
\sigma_{t o t}^{\mathrm{ND}}=2 \sigma_{t o t}^{\mathrm{NN}}+\delta \sigma_{t o t}
$$

where

$$
\delta \sigma_{t o t}=\frac{2}{k^{2}} \int d^{2} q^{\prime} S\left(\mathbf{q}^{\prime}\right) f_{k}\left(\mathbf{q}^{\prime}\right) f_{k}\left(-\mathbf{q}^{\prime}\right) .
$$

This correction term represents rescattering events.

Adding the rescattering corrections to the differential cross section for elastic scattering leads to

$$
\begin{aligned}
\frac{d \sigma^{\mathrm{ND}}}{d \Omega_{l}} & =4 S^{2}\left(\frac{1}{2} \mathbf{q}\right)\left|f_{k}(\mathbf{q})\right|^{2} \\
& -\frac{2}{\pi k} S\left(\frac{1}{2} \mathbf{q}\right) \Im m\left[f_{k}^{*}(\mathbf{q}) \int d^{2} q^{\prime} S\left(\mathbf{q}^{\prime}\right) f_{k}\left(1 / 2 \mathbf{q}+\mathbf{q}^{\prime}\right) f_{k}\left(1 / 2 \mathbf{q}-\mathbf{q}^{\prime}\right)\right] \\
& +\left(\frac{1}{2 \pi k}\right)^{2}\left|\int d^{2} q^{\prime} S\left(\mathbf{q}^{\prime}\right) f_{k}\left(1 / 2 \mathbf{q}+\mathbf{q}^{\prime}\right) f_{k}\left(1 / 2 \mathbf{q}-\mathbf{q}^{\prime}\right)\right|^{2} .
\end{aligned}
$$

Next, we need to discuss the choice of the two-particle scattering amplitude $f_{k}(\mathbf{q})$. In the original work of Refs. 11, 25, 26], the eikonal phase is given in terms of the potential $V(\mathbf{r})$ as

$$
\begin{aligned}
\chi(\mathbf{b}) & =-\frac{1}{v} \int_{-\infty}^{\infty} V(\mathbf{b}+\mathbf{z}) d z \\
& =-\frac{2 m}{k} \int_{0}^{\infty} V\left(\sqrt{b^{2}+z^{2}}\right) d z
\end{aligned}
$$

Where $\mathbf{z}$ is a vector parallel to $\mathbf{k}$. The above definition would result in a two-body scattering amplitude in the eikonal approximation. Studies of the quality of the eikonal approximation for potential scattering have been carried out in detail in the past for different potentials. The for us interesting case of Yukawa-type potentials is considered e.g. in Refs. [27, 28]. Since our main interest is the comparison of the $n+d$ cross sections, we need to use the same two-particle scattering amplitude $f_{k}(\mathbf{q})$ for our Glauber calculation as we use for the Faddeev calculation. Thus we need to use the scattering amplitude obtained from the solution of a Lippmann-Schwinger equation with the potential $V$ as driving term.

This two-particle scattering amplitude $f_{c}\left(\mathbf{p}^{\prime}, \mathbf{p}\right)$ is given in the c.m. frame and is related to the on-shell two-body $t$-matrix as

$$
\left\langle\mathbf{p}^{\prime}|V| \psi_{\mathbf{p}}^{(+)}\right\rangle=\left\langle\mathbf{p}^{\prime}\right| t\left(E_{p}=\frac{p^{2}}{m}|\mathbf{p}\rangle \equiv t\left(\mathbf{p}^{\prime}, \mathbf{p} ; \frac{p^{2}}{m}\right),\right.
$$

and leads to the scattering amplitude

$$
f_{c}\left(\mathbf{p}^{\prime}, \mathbf{p}\right)=-\frac{m}{2}(2 \pi)^{2} t\left(\mathbf{p}^{\prime}, \mathbf{p} ; \frac{p^{2}}{m}\right) .
$$

For a scalar potential $t$ is also a scalar and depends on the magnitude of $|\mathbf{p}|=\left|\mathbf{p}^{\prime}\right|$ and the angle $x_{c}=\hat{\mathbf{p}^{\prime}} \cdot \hat{\mathbf{p}}$ between the two vectors $\mathbf{p}^{\prime}$ and $\mathbf{p}[29]$,

$$
t\left(\mathbf{p}^{\prime}, \mathbf{p} ; \frac{p^{2}}{m}\right)=t\left(p, p, x_{c} ; \frac{p^{2}}{m}\right) \equiv t\left(p, x_{c}\right) .
$$


For identical particles we use as in the Faddeev calculations the symmetrized $t$-matrix $t_{s}\left(\mathbf{p}^{\prime}, \mathbf{p} ; \frac{p^{2}}{m}\right)$. The c.m. differential cross section for the scattering of two identical particles in then given as

$$
\frac{d \sigma}{d \Omega_{c}}=\left|f_{c}^{s}\left(p, x_{c}\right)\right|^{2}
$$

and the laboratory differential cross section as

$$
\frac{d \sigma}{d \Omega_{l}}=4 x_{l} \frac{d \sigma}{d \Omega_{c}},
$$

where the standard relation between c.m. and laboratory scattering angles for two equal mass particles is given as

$$
\begin{aligned}
x_{l} \equiv \hat{\mathbf{k}} \cdot \hat{\mathbf{k}^{\prime}} & =\sqrt{\frac{x_{c}+1}{2}} \\
x_{c} & =2 x_{l}^{2}-1 .
\end{aligned}
$$

If one defines

$$
\frac{d \sigma}{d \Omega_{l}}=\left|f_{l}^{s}\left(k, x_{l}\right)\right|^{2}
$$

the two-particle scattering amplitude in the laboratory frame reads

$$
f_{l}^{s}\left(k, x_{l}\right)=2 \sqrt{x_{l}} f_{c}^{s}\left(p, x_{c}\left(x_{l}\right)\right) .
$$

All that remains now is choosing the unit vector $\hat{n}$. The original suggestion by Glauber [1, 26] is

$$
\text { (a) : } \hat{n}=\hat{\mathbf{k}} \text {, }
$$

with the approximation $|\mathbf{k}|=\left|\mathbf{k}^{\prime}\right|$. In other works [25, 27, 28] the choice

$$
\text { (b) }: \hat{n}=\widehat{\mathbf{k}+\mathbf{k}^{\prime}}
$$

is preferred. We will use both choices in our calculations to explore the differences. The momentum transfer $\mathbf{q}$ enters the scattering amplitude $f_{c}$ in the form of

$$
x_{c}=1-\frac{\mathbf{q}^{2}}{2 p^{2}}
$$

The Glauber ansatz requires to replace the momentum transfer $\mathbf{q}^{2}$ by $\mathbf{q}_{\perp}{ }^{2}$ with $\mathbf{q}_{\perp}$ being orthogonal to $\hat{n}$. For the two cases of Eqs. (3.24) and (3.25) this leads to

$$
\begin{aligned}
& \text { (a) : } \mathbf{q}_{\perp}{ }^{2}=k^{2}\left(1-x_{l}^{2}\right) \\
& \text { (b) : } \mathbf{q}_{\perp}{ }^{2}=k^{2}\left(1-x_{l}^{2}\right) \frac{4 x_{l}^{2}}{1+3 x_{l}^{2}} .
\end{aligned}
$$

For forward angles both choices become equivalent.

The total cross section for two-body scattering entering into Eq. (3.10) is then explicitly given as

$$
\sigma_{\text {tot }}^{\mathrm{NN}}=\int d \Omega_{c}\left|f_{c}^{s}\left(p, x_{c}\right)\right|^{2} .
$$

The scattering amplitude $f_{k}(\mathbf{q})$ entering Eq. (3.11) can now be directly obtained from Eq. (3.23) as

$$
f_{k}(\mathbf{q}) \rightarrow f_{l}^{s}\left(k, x_{l}\right) .
$$

In calculating the angle $x_{c}\left(x_{l}\right)$, the momentum transfer of Eq. (3.26) must then be replaced by $q_{\perp}^{2}$, and $x_{l}$ is related to $x_{c}$ through Eq. (3.21).

For the second order correction to the differential cross section the integral in Eq. (3.14) needs to be evaluated. According to the Glauber ansatz the argument of the scattering amplitude needs to be evaluated as $1 / 2 \mathbf{q}_{\perp} \pm \mathbf{q}^{\prime}$, so that the integration is carried out in the plane defined by $\hat{n}$. Explicitly, the integral is calculated as

$$
\int d^{2} q^{\prime} \quad S\left(q^{\prime}\right) f_{k}^{s}\left(\frac{1}{2} \mathbf{q}+\mathbf{q}^{\prime}\right) f_{k}^{s}\left(\frac{1}{2} \mathbf{q}-\mathbf{q}^{\prime}\right) \rightarrow
$$




$$
4\left(\frac{m}{2}\right)^{2}(2 \pi)^{4} \int_{0}^{q_{\max }} d q^{\prime} q^{\prime} S\left(q^{\prime}\right) \int_{0}^{2 \pi} d \phi \sqrt{x_{l}\left(x^{(+)}\right) x_{l}\left(x^{(-)}\right)} t_{s}\left(p, x^{(+)}\right) t_{s}\left(p, x^{(-)}\right),
$$

with the definition of $t_{s}(p, x)$ from Eq. (3.18) and

$$
x^{( \pm)}=1-\frac{1 / 4 q_{\perp}^{2} \pm q_{\perp} q^{\prime} \cos \phi+q^{\prime 2}}{2 p^{2}} .
$$

Here $\phi$ is the angle between the vectors $\mathbf{q}_{\perp}$ and $\mathbf{q}^{\prime}$. The upper limit $q_{\max }$ is the maximum momentum transfer allowed for a given projectile laboratory energy, corresponding to $x_{c}=-1$ in the angle argument of the $t$-matrix.

For the second order correction to the total cross section, Eq. (3.12), we only need to consider the special case $q_{\perp}=0$. Then the integral of Eq. (3.30) simplifies to

$$
\delta \sigma_{t o t}=\frac{2}{k^{2}} m^{2}(2 \pi)^{5} \int_{0}^{q_{\max }} d q^{\prime} q^{\prime} S\left(q^{\prime}\right) x_{l}(x) \Re e\left[t_{s}^{2}(p, x)\right],
$$

with $x=1-\frac{q^{\prime 2}}{2 p^{2}}$.

In Ref. [26] a further approximation is suggested arguing that if the form factor of the deuteron decreases much more rapidly than the scattering amplitude as function of $q^{\prime}$, then the integral in Eq. (3.32) may be approximated by

$$
\delta \sigma_{t o t}=\frac{2}{k^{2}} m^{2}(2 \pi)^{5} \Re e\left[t_{s}^{2}(p, x=1)\right] \int_{0}^{q_{\max }} d q^{\prime} q^{\prime} S\left(q^{\prime}\right),
$$

where the scattering amplitude in forward direction $(x=1$ or $\theta=0)$ is taken out of the integral.

\section{RESULTS AND DISCUSSION}

Our explicit calculations are based on an interaction chosen as superposition of two Yukawa interactions of the Malfliet-Tjon [30] type,

$$
V\left(\mathbf{p}^{\prime}, \mathbf{p}\right)=\frac{1}{2 \pi^{2}}\left(\frac{V_{R}}{\left(\mathbf{p}^{\prime}-\mathbf{p}\right)^{2}+\mu_{R}^{2}}-\frac{V_{A}}{\left(\mathbf{p}^{\prime}-\mathbf{p}\right)^{2}+\mu_{A}^{2}}\right),
$$

in which the parameters, given in Table \, are fitted such that a bound state, the 'deuteron' is supported with a binding energy $E_{d}=-2.23 \mathrm{MeV}$. This interaction enters the nonrelativistic Faddeev equation for identical bosons via the symmetrized two-body $t$-matrix. The Faddeev equation is exactly solved without a partial wave decomposition, using momentum vectors and angles between them. This allows us to calculate three-body observables in the $\mathrm{GeV}$ region. The details of the calculations are given in Ref. [17]. The deuteron wave function entering the form factor of Eq. (3.7) is obtained as solution of a bound state equation with the potential of Eq. (4.1) as input. As a note, we are of course aware that this is a model study, in which the two-body $t$-operators are generated through a non-relativistic Lippmann Schwinger equation from a simple Hermitian two-body force.

\section{A. Total Cross Sections}

First we want to explore the convergence of the Faddeev multiple scattering series when considering total cross sections. The top panel of Fig. [1 shows the total cross section for three-body scattering calculated using Eq. (2.7) as function of the order in the two-body $t$-matrix when adding up successively the terms of the multiple scattering series created by Eq. (2.1). The convergence is shown for selected projectile laboratory energies ranging from $200 \mathrm{MeV}$ to $2 \mathrm{GeV}$. For energies larger than $1 \mathrm{GeV}$ the curves are essentially flat, meaning that the first order term given by $T=t P$ is already sufficient to capture the result of a full Faddeev calculation. At $500 \mathrm{MeV}$ one needs at least one iteration (or one rescattering term) to reach the exact Faddeev result, whereas at $200 \mathrm{MeV}$ one needs more terms, which however give small contributions. The middle part of the panel shows the total cross section for elastic scattering and the bottom one the total cross section for breakup reactions. While the total cross section for elastic scattering converges very fast for projectile energies above $500 \mathrm{MeV}$, the one for breakup reactions requires at least one rescattering term even in the $\mathrm{GeV}$ range.

Our Glauber calculation contains only a first order term and a rescattering correction. First, we concentrate on the total cross section and compare each order separately with the corresponding order of the Faddeev calculation. 
The first order terms are compared in Fig. 2 as function of the projectile laboratory energy. According to Eq. (3.10) the first order contribution to the total $n+d$ cross section is simply given by twice the two-body total cross section. From Fig. 2 we see that for energies greater than $200 \mathrm{MeV}$ both first order terms agree. This is not surprising, since the first order Faddeev term, $T=t P$, contains twice the two-body t-matrix, the permutation operator guarantees scattering contributions from both constituents of the target. Our Glauber calculations start from the same two-body $t$-matrix as the Faddeev calculation. From this point of view it is not surprising that both first order calculations agree so well. The same conclusion was already reached in Ref. 24].

A more crucial test is a comparison of the second order correction term. In Fig. 3 we show the first rescattering correction to the $n+d$ total cross section, which corresponds to second order contribution in $t$ of the Faddeev multiple scattering series. The Faddeev result is shown as solid line. A first observation is that this rescattering term contributes significantly at lower energies, and is still present at $2 \mathrm{GeV}$. Thus, the very naive expectation that the $n+d$ total cross section comprises only the scattering from the target constituents is not fulfilled in the energy regime up to $2 \mathrm{GeV}$ which is considered here. There is always a contribution due to rescattering. The second order Glauber correction $\delta \sigma$ from Eqs. (3.13) and (3.30) is given by the short dashed line. Here we see that in contrast to the first order contribution the two lines only start to agree around $1 \mathrm{GeV}$. This may be understood when having in mind that the second order Faddeev term is explicitly given as $t P G_{0} t P$, where the free propagator $G_{0}$ describes the arbitrary free motion of the three particles in the intermediate state. The Glauber second order term assumes that the target constituents are frozen. At sufficiently high energy this assumptions seems to be sufficient to describe the rescattering within the target, which sometimes is referred to as 'shadowing' correction.

Franco and Glauber [26] suggest a further approximation to $\delta \sigma$. They argue that if the deuteron form factor decreases much more rapidly than the scattering amplitude as function of the integration variable $q^{\prime}$, then the integral of Eq. (3.32) may be approximated by the expression of Eq. (3.33), where the scattering amplitude is approximated by its value in forward direction and taken out of the integral. The double-dash-dotted curve of Fig. 3 represents this approximation. It is quite obvious that this approximation is too simplistic and gives a second order contribution almost double the size of the original correction at all energies considered. The long-dashed curve represents the analytic evaluation of the second order Faddeev term in the optical theorem of Eq. (2.9). The contribution calculated in there overestimates the second order contribution in a similar fashion. In Ref. 24], integrals over the two-body $t$-matrix and the deuteron wave function were evaluated using the method of steepest descent. This is equivalent to extracting the dominant part of an integral as a constant from the integral. The failure of those two approximations should lead to the conclusion, that although it is true that for higher energies the scattering amplitude is peaked in forward direction [29], the integration over the form factor is important and should not be approximated further.

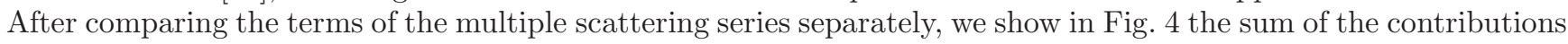
as function of the projectile laboratory energy. The solid line represents the exact Faddeev calculation, in which the multiple scattering series is summed to all orders. As reference, the dotted line represents twice the two-body total cross section $2 \sigma^{\mathrm{NN}}$, to which the different second order contributions shown in Fig. 3 are added. The Glauber calculations up to second order agrees with the full Faddeev result starting from about $1 \mathrm{GeV}$. Both, the simplification of the Glauber second order term, Eq. (3.33), and the high energy limit of Eq. (2.9) considerably overestimate the exact Faddeev result at all energies considered.

\section{B. Differential Cross Sections}

Similar to the investigations of the total cross section, we want to start comparing the first order calculations of the differential cross section at various energies. The differential cross section for elastic $n+d$ scattering is calculated from the operator $U$ for elastic scattering, Eq. (2.3), and given as function of the laboratory solid angle as

$$
\frac{d \sigma^{\mathrm{ND}}}{d \Omega_{l}}=(2 \pi)^{4} \frac{2}{9} m^{2} \frac{\left(x_{l}+\sqrt{x_{l}^{2}+3}\right)^{2}}{\sqrt{x_{l}^{2}+3}}\left|\left\langle\phi_{d} \hat{q} q_{0}|U| \phi_{d} \mathbf{q}_{\mathbf{0}}\right\rangle\right|^{2} .
$$

Here $x_{l}=\cos \theta_{l} \equiv \hat{\mathbf{k}}^{\prime} \cdot \hat{\mathbf{k}}$ represent the laboratory scattering angle for the $n+d$ system, and $\mathbf{q}_{0}$ its c.m. momentum. For calculating the Glauber differential cross section from Eq. (3.11), we have to consider the projection of the momentum transfer $\mathbf{q}$ on to a plane either perpendicular to the laboratory momentum $\mathbf{k}$ or to the sum of incoming and outgoing momenta $\mathbf{k}+\mathbf{k}^{\prime}$, as indicated in Eqs. (3.24) and (3.25). Since both choices are employed in the literature, we will consider both in this study.

Taking the considerations from the previous subsection as guidance, we start our comparison at $500 \mathrm{MeV}$ projectile energy and show in Fig. 5 the first order calculations for the differential cross section as function of the laboratory scattering angle. The solid line represents the first order Faddeev calculation, and the dashed and dash-dotted lines the first order Glauber calculations with the choices (a), Eq. (3.24), and (b), Eq. (3.25), for the projection of the 
momentum transfer. Both choices lead to identical results for small scattering angles. The first small deviation can be seen around the first diffraction minimum. Though the Glauber approximation should only be valid in forward direction, we plot the entire allowed angle region in order to obtain quantitative insights in the region of angular validity. Both Glauber calculations start to deviate from the Faddeev first order calculation as well as from each other at $\theta_{l} \approx 50^{\circ}$, which corresponds to a momentum transfer of about $740 \mathrm{MeV}$. For comparison, we also add the fully converged Faddeev result as dash-double-dotted line to the figure. An obvious difference between the first order and the fully converged Faddeev calculations is the first diffraction minimum, which is filled in by rescattering corrections. Rescattering contributions are also important for the large angle (high momentum transfer) behavior of the differential cross section [17, 21].

Fig. 6 shows the differential cross section for similar calculations at the considerably higher energy of $1.5 \mathrm{GeV}$. Again, the first order Faddeev calculation and the first order Glauber calculations with the two different choices of $\mathbf{q}_{\perp}$ are close to each other up to $\theta_{l} \approx 50^{\circ}$, which now corresponds to a momentum transfer of about $1250 \mathrm{MeV}$. However, the first order calculations differ from the fully converged Faddeev calculation already at much smaller angles, around $\theta_{l} \approx 12^{\circ}$, which corresponds to roughly $350 \mathrm{MeV}$ in the momentum transfer. While the Glauber formulation is intended as high energy approximation, we are interested in exploring how the differential cross sections of the first order Glauber and Faddeev calculations compare at lower energies. In Figs. 7 and 8 the differential cross sections for laboratory projectile energies of 200 and $100 \mathrm{MeV}$ are displayed. For $200 \mathrm{MeV}$ the two different choices of $\mathbf{q}_{\perp}$ in the Glauber first order calculations already differ at $\theta_{l} \approx 25^{\circ}$, corresponding to a momentum transfer of about $270 \mathrm{MeV}$. At roughly the same angle the first order Faddeev calculations also deviates from the converged solution. It is well known that the Faddeev multiple scattering series converges very slowly at such low energies [23], so this finding is not surprising. However, at very small angles, all calculations shown roughly agree. This becomes different, if one looks at the even lower energy of $100 \mathrm{MeV}$ laboratory projectile energy. Here the first order Glauber calculations do not agree with the first order Faddeev calculation even at small angles. It is also very obvious that at this low energy the first order Faddeev calculations is quite different from the fully converged one. It is however noteworthy, that both first order Glauber calculations are closer to the converged Faddeev result in forward direction than is the calculation based on the first order Faddeev term. The two different choices for $\mathbf{q}_{\perp}$ start to differ around $\theta_{l} \approx 25^{\circ}$, which corresponds now only to a momentum transfer of roughly $180 \mathrm{MeV}$. The overall observation is that at such a low energy neither a first order Faddeev calculation nor a Glauber calculation are a good representation of the fully converged Faddeev result.

Next we add the Glauber rescattering correction of Eq. (3.14) to the differential cross section. In Fig 9 we show the effect of this term on the differential cross section at $500 \mathrm{MeV}$ laboratory projectile energy together with the contribution of the second order Faddeev rescattering term. While the Faddeev first order rescattering term gives a large contribution to the first minimum, the Glauber rescattering term fails to do this, independent of the choice of the unit vector $\hat{n}$. Obviously, the minima are more sensitive to the interference of the two terms of the scattering amplitude. Thus here the very different structure of the Faddeev and Glauber rescattering terms are clearly visible. It should also be noted that in the first minimum one Faddeev rescattering term is already sufficient to coincide with the fully converged Faddeev calculation. At slightly larger angles more rescattering terms are required in the Faddeev calculation to reach convergence.

This general feature of the difference between the two approaches does not change with increasing energy. In Fig 10 the differential cross section is shown for a laboratory projectile energy of $1 \mathrm{GeV}$. While the total cross section for both, Faddeev and Glauber calculations started to agree at energies of $1 \mathrm{GeV}$ and higher when adding the second order correction term in both schemes, this is not the case for the differential cross section. The second order Faddeev rescattering term again gives a much larger contribution in the first minimum than the Glauber rescattering correction. This trend continues up to $2 \mathrm{GeV}$, the highest energy we consider here. We also can observe that with increasing energy the difference between the two choices of $\hat{n}$ decreases. To investigate at which energies there are differences, we need to consider lower projectile energies. In Fig. 11] the differential cross section for $200 \mathrm{MeV}$ projectile laboratory energy is shown. Here we can first observe, that the second order Glauber correction also increases the cross section in the first minimum, but again by far less than the Faddeev rescattering term does. We also observe a distinct difference between the two choices of $\hat{n}$. At $200 \mathrm{MeV}$ the choice (a) almost has no effect at larger angles, whereas choice (b) shows a big contribution. However, since the Glauber expression should be valid only in forward direction, one should not put any physical relevance to this difference.

After having seen that the Glauber rescattering term does not compare well with the corresponding Faddeev term in diffraction minima, we want to consider the differential cross section in the very forward direction. This is the physical regime for which the Glauber ansatz was developed and where it should perform best. In Fig. 12 we show the three-body differential cross section for very forward angles for laboratory projectile energies of $100 \mathrm{MeV}, 200 \mathrm{MeV}$, $500 \mathrm{MeV}$, and $800 \mathrm{MeV}$. We again compare the results from the first and second order terms in the Faddeev multiple scattering series to the fully converged Faddeev calculation, and the first order Glauber calculation with its rescattering correction to the Faddeev calculations. Both choices of $\hat{n}$ coincide for very small angles. Thus we arbitrarily choose 
(b) for the Glauber calculations of Fig. 12 Since we already established from the comparison of the total cross sections, that the Glauber calculations do very well for energies of $1 \mathrm{GeV}$ and higher, we concentrate here on low and intermediate range energies. At $800 \mathrm{MeV}$ essentially all calculations agree with each other in the very forward direction. At $500 \mathrm{MeV}$ the first order Faddeev term slightly over-predicts the fully converged result. The first Faddeev rescattering term has almost no effect, and one needs higher order rescattering contributions to achieve a converged result. The Glauber first order calculation differs from the Faddeev first order and only slightly over-predicts the converged Faddeev result. The contribution of the Glauber rescattering term is small, and lowers the cross section for the very forward angles directly to the full Faddeev result. The situation at $200 \mathrm{MeV}$ is similar. Again, the first order Faddeev and Glauber calculations differ, and the Glauber calculation already coincides with the fully converged Faddeev result. The Glauber rescattering contribution again slightly lowers the cross section in forward direction. At this energy it is interesting to observe that the first rescattering contribution of the Faddeev multiple scattering series increases the cross section in forward direction. To reach convergence eight terms of the multiple scattering series must be summed up at this energy. At $100 \mathrm{MeV}$ the Glauber first order calculation is already quite close to the fully converged Faddeev results, in contrast to the first order Faddeev calculation. The Glauber rescattering correction then lowers the cross section essentially to the exact Faddeev result. Thus, for the differential cross section in very forward direction the Glauber calculation corrected by the rescattering term captures the exact Faddeev result already at $100 \mathrm{MeV}$. Fig. 8 shows that this good agreement is valid to about $\theta_{l} \approx 20^{\circ}$.

The behavior of the differential cross section in the extreme forward direction appears in a very similar fashion in the total cross section for elastic scattering, $\sigma_{e l}$, which is obtained by integrating over the differential cross section. Table II lists the total cross sections for elastic scattering as function of the laboratory projectile energy for the converged Faddeev calculations, Faddeev calculations including only the first two rescattering terms as well as the Glauber calculations. We see that already from $200 \mathrm{MeV}$ on the first order Glauber calculations match the converged Faddeev results reasonably well. The Glauber rescattering correction is quite small, and always lowers the cross section, whereas the first Faddeev rescattering correction increases the cross section, and only the second rescattering correction (3rd order in the multiple scattering series) lowers it. It is also obvious from the table that at $100 \mathrm{MeV}$ many more terms of the Faddeev multiple scattering series are necessary to obtain a converged total cross section. The Glauber result with rescattering correction is similar in size to the Faddeev result with two rescattering corrections, though this is most likely accidental. A Glauber calculation for the total cross section for elastic scattering at $100 \mathrm{MeV}$ is not trustworthy anymore, since the differential cross sections do not really agree.

The total cross section is the sum of the total cross sections for elastic scattering and breakup reactions, $\sigma_{t o t}^{\mathrm{ND}}=$ $\sigma_{e l}^{\mathrm{ND}}+\sigma_{b r}^{\mathrm{ND}}$. In Refs. [17, 21] this relation was used to estimate the quality of the numerical solutions of the Faddeev equation. In a Faddeev calculation the total cross section for breakup reactions is obtained by integration over the five-fold differential cross section for breakup, i.e. by summing over all possible breakup configurations. Here we can employ this relation to obtain a total cross section for breakup reactions within the Glauber framework. This is done in Table III] The left side lists the Faddeev total cross sections for elastic scattering and breakup reactions with their sum, the total cross section, as function of laboratory projectile energy. The right side lists the Glauber total cross section obtained from Eq. (3.12). The Glauber total cross sections for breakup reactions are obtained by subtracting the Glauber total cross sections for elastic scattering from Table凹from these numbers. For energies less than $500 \mathrm{MeV}$ the so obtained breakup cross sections are definitely not competitive with the Faddeev results. However, at $1.5 \mathrm{GeV}$, the Glauber breakup cross section starts to match the Faddeev breakup cross section within 5\%. Though this finding may look surprising, it could indicate that at sufficiently high energy the dominant ejectiles of a breakup reaction exit into the forward cone. That region of phase space however is the one, for which the Glauber ansatz was designed. Thus it seems that for $\mathrm{GeV}$ projectile energies obtaining a total cross section for breakup reactions from the Glauber total and elastic cross section via the optical theorem captures the overall breakup reaction. Of course the description of detailed exclusive $n+d$ observables will never be accessible in the Glauber formulation.

\section{SUMMARY AND CONCLUSIONS}

In this study we perform fully converged Faddeev calculations for three identical bosons (our model for the $n+d$ system) in the intermediate energy regime between $100 \mathrm{MeV}$ and $2 \mathrm{GeV}$. We calculate total cross sections as well as differential cross sections for elastic scattering. The key point of those calculations is the use of vector momenta in the formulation, so that all partial waves are automatically included. We then calculated the same cross sections using Glauber formulation [25, 26, 27]. However a key difference to this early work is that the two-body scattering amplitude entering our Glauber expressions is the solution of a two-body Lippmann-Schwinger equation, i.e. the same input as is used in the Faddeev calculations. As two-body interaction we employ a superposition of two Yukawa terms, one attractive, the other repulsive, for which the parameters are chosen such that a bound state at the empirical deuteron binding energy is supported. The deuteron wave function and form factor are also calculated from this interaction. 
By using the identical two-body input for the Faddeev and Glauber calculations we make sure that both calculations are based on the same ingredients. This way we can clearly attribute differences in the observables to the different treatment of multiple scattering in the two formulations. The first order term in the Glauber formulation is given by twice the two-body scattering amplitude, which is folded with the deuteron form factor to give the differential cross section and leads to $2 \sigma^{\mathrm{NN}}$ for the total cross section. The first order Faddeev term is given by $T=t P$, thus having a similar structure. Comparing the total cross sections obtained from the first order term leads to the conclusion that from projectile energies of about $200 \mathrm{MeV}$ onward both calculations agree. For the differential cross section agreement to about $\theta_{l} \approx 45^{\circ}$ is achieved for projectile energies of $500 \mathrm{MeV}$ and higher. For $200 \mathrm{MeV}$ the angular range of agreement is already less, and at $100 \mathrm{MeV}$ the first order calculations do not agree with each other. In general we can conclude, that in first order the Glauber and Faddeev calculations for observables considered, total and differential cross sections for elastic scattering are remarkably close for energies higher than $200 \mathrm{MeV}$ projectile energy.

The consideration of the first rescattering correction in both formulation shows that there is a distinct dependence on the observables considered. As a reminder, the Glauber formulation leads only to one rescattering correction, whereas the Faddeev formulation as integral equation has an in principle infinite series of rescattering corrections. As far as the correction to the total cross section is concerned, the contribution from the first Glauber and Faddeev rescattering term starts to become close at $500 \mathrm{MeV}$ and is identical in size at $1 \mathrm{GeV}$. We also want to point out, that this rescattering correction to the total cross section, the shadowing (better named anti-shadowing here, since the contribution increases the cross section), is still present at $2 \mathrm{GeV}$, the highest energy we considered. Thus we conclude, that for a good description of the total cross section in the intermediate energy regime the consideration of the second order correction in the Glauber as well as in the Faddeev formulation is essential.

For the differential cross section for elastic scattering the conclusions are twofold. First, when considering the very forward direction, we find that at projectile energies of 100 and $200 \mathrm{MeV}$ the second order correction brings the Glauber calculation of the differential cross section in quite good agreement with the result of the fully converged Faddeev calculation. In contrast, the first Faddeev rescattering term even has the opposite effect and moves the cross section away from the converged calculation. At projectile energies of $500 \mathrm{MeV}$ and higher, the second order corrections in the very forward direction are extremely small and the first order Faddeev and Glauber calculations already agree quite well with each other and the converged Faddeev calculation. A similar conclusion can be drawn when considering the total cross sections for elastic scattering. Second, when concentrating on the diffractive structure of the differential cross section given in our model, we see distinct differences between the behavior of the Faddeev and Glauber second order correction. Whereas the Faddeev correction fills in the minima, the Glauber correction can not do that. A similar observation was made in Ref. [16] in the context of proton elastic scattering from halo nuclei considering energies from 100 to $200 \mathrm{MeV}$ per nucleon. However we find that even at much higher energies the Glauber second order correction does not come close to the effect of the Faddeev correction. For energies higher than $500 \mathrm{MeV}$ the first Faddeev rescattering correction already captures the bulk of rescattering corrections and coincides with the converged calculation in the first minimum. Diffraction minima are well known to be sensitive to the dynamics of the system or interference effects. The assumption of fixed target particles leading to the specific form of the Glauber rescattering correction proves to be too simple to capture the much more involved structure of the first Faddeev rescattering term. We also need to point out here, that the diffractive structures shown in the differential cross sections of this work are a result of our underlying assumption of identical bosons. The true $n+d$ differential cross section has only one minimum and one might speculate that a Glauber calculation at higher energies including the rescattering term and being based on nucleon-nucleon (NN) interactions describing the NN observables has a chance to describe the $n+d$ total cross and possibly the differential cross section certainly in forward direction quite well, provided a relativistic formulation is going to be used.

\section{Acknowledgments}

This work was performed in part under the auspices of the U. S. Department of Energy, Office of Nuclear Physics under contract No. DE-FG02-93ER40756 with Ohio University, and in part under NSF grant PHY-0653312 with the Ohio State University.

We thank the Ohio Supercomputer Center (OSC) for the use of their facilities under grant PHS206. In addition we appreciate helpful comments from David R. Harrington. Ch.E. thanks the nuclear theory group of the Ohio State 
University for their warm hospitality during the time this work was carried out.

[1] R.J. Glauber, Phys. Rev. 100, 242 (1955).

[2] R.J. Glauber in Lectures in Theoretical Physics, edited by W. Brittin and L.G. Dunham (Interscience, New York, 1959), Vol. 1.

[3] C. Ciofi degli Atti, L.P. Kaptari, Phys. Rev. C66, 044004 (2002).

[4] R. Schiavilla, O. Benhar, A. Kievsky, L. E. Marcucci and M. Viviani, Phys. Rev. C72, 064003 (2005) arXiv:nucl-th/0508048.

[5] A. Bianconi, S. Jeschonnek, N. N. Nikolaev and B. G. Zakharov, Phys. Lett. B343, 13 (1995); A. Bianconi, S. Jeschonnek, N. N. Nikolaev and B. G. Zakharov, Phys. Rev. C 53, 576 (1996). A. Bianconi, S. Jeschonnek, N. N. Nikolaev and B. G. Zakharov, Nucl. Phys. A608, 437 (1996).

[6] L. Frankfurt, G. A. Miller and M. Strikman, arXiv:0803.4012 [nucl-th]; L. Frankfurt, M. Strikman and M. Zhalov, Phys. Lett. B 503, 73 (2001) arXiv:hep-ph/0011088.

[7] O. Benhar, S. Fantoni, N. N. Nikolaev, J. Speth, A. A. Usmani and B. G. Zakharov, J. Exp. Theor. Phys. 83, 1063 (1996) [Zh. Eksp. Teor. Fiz. 110, 1933 (1996)] arXiv:nucl-th/9608016]; N. N. Nikolaev, A. Szczurek, J. Speth, J. Wambach, B. G. Zakharov and V. R. Zoller, Nucl. Phys. A 567, 781 (1994) arXiv:nucl-th/9303006.

[8] L. Frankfurt, M. Strikman and M. Zhalov, Phys. Rev. C67, 034901 (2003) arXiv:hep-ph/0210303]; V. Guzey and M. Strikman, arXiv:0804.4232 [hep-ph].

[9] M. M. Sargsian, Int. J. Mod. Phys. E 10, 405 (2001) arXiv:nucl-th/0110053; M. M. Sargsian, T. V. Abrahamyan, M. I. Strikman and L. L. Frankfurt, Phys. Rev. C71, 044614 (2005) arXiv:nucl-th/0406020] ․ L. Frankfurt, M. M. Sargsian and M. I. Strikman, Phys. Rev. C56, 1124 (1997) arXiv:nucl-th/9603018.

[10] C. C. degli Atti and L. P. Kaptari, Phys. Rev. C71, 024005 (2005).

[11] B. Van Overmeire, W. Cosyn, P. Lava and J. Ryckebusch, Phys. Rev. C73, 064603 (2006). arXiv:nucl-th/0603013.

[12] J. Ryckebusch, D. Debruyne, P. Lava, S. Janssen, B. Van Overmeire, T. Van Cautern, Nucl. Phys. A 728, 226 (2003).

[13] J.S. Al-Khalili, J.A. Tostevin, I.J. Thompson, Phys. Rev. C54, 1843 (1996).

[14] J.S. Al-Khalili, J.A. Tostevin, Phys. Rev. C57, 1846 (1998).

[15] A. Bianconi and M. Radici, Phys. Rev. C53, 563 (1996). arXiv:nucl-th/9510063; A. Bianconi and M. Radici, Phys. Lett. B 363, 24 (1995). arXiv:nucl-th/9509045.

[16] R. Crespo, E. Cravo, A. Deltuva, M. Rodriguez-Gallardo and A. C. Fonseca, Phys. Rev. C76, 014620 (2007).

[17] H. Liu, Ch. Elster and W. Glöckle, Phys. Rev. C 72, 054003 (2005).

[18] H. Liu, Ch. Elster and W. Gloeckle, Few Body Syst. 33, 241 (2003).

[19] T. Lin, Ch. Elster, W. N. Polyzou and W. Glöckle, Phys. Rev. C 76, 014010 (2007).

[20] T. Lin, Ch. Elster, W. N. Polyzou and W. Glöckle, Phys. Lett. B 660, 345 (2008).

[21] T. Lin, Ch. Elster, W. N. Polyzou, H. Witała and W. Glöckle, arXiv:0801.3210] [nucl-th].

[22] W. Glöckle, The Quantum Mechanical Few-Body Problem (Springer-Verlag, Berlin-Heidelberg, 1983).

[23] W. Glöckle, et. al., Phys. Rep. 274, 107 (1996).

[24] Ch. Elster, W. Schadow, H. Kamada and W. Glöckle, Phys. Rev. C58, 3109 (1998).

[25] David R. Harrington, Phys. Rev. 135, B358 (1964).

[26] V. Franco and R.J. Glauber, Phys. Rev. 42, 195 (1966).

[27] F.W. Byron, C.J. Joachain, E.H. Mund, Phys. Rev. D8, 2622 (1973).

[28] S.J. Wallace, Ann. Phys. (N.Y.), 78, 190 (1973).

[29] Ch. Elster, J.H. Thomas, and W. Glöckle, Few-Body Systems 24, 55 (1998).

[30] R. A. Malfliet and J. A. Tjon Nucl. Phys. A127,161(1969). 


\begin{tabular}{|c|c|c|c|c|}
\hline$V_{A}[\mathrm{MeV} \mathrm{fm}]$ & $\mu_{A}\left[\mathrm{fm}^{-1}\right]$ & $V_{R}[\mathrm{MeV} \mathrm{fm}]$ & $\mu_{R}\left[\mathrm{fm}^{-1}\right]$ & $E_{d}[\mathrm{MeV}]$ \\
\hline 626.8932 & 1.55 & 1438.7228 & 3.11 & -2.23 \\
\hline
\end{tabular}

TABLE I: The parameters and deuteron binding energy for the Malfliet-Tjon type potential (MT3) of our calculation. As conversion factor We use units such that $\hbar c=197.3286 \mathrm{MeV} \mathrm{fm}=1$.

\begin{tabular}{|c|ccc|c||cc|cc|}
\hline & \multicolumn{4}{|c|}{ Faddeev } & \multicolumn{2}{c|}{ Glauber (a) } & \multicolumn{2}{c|}{ Glauber (b) } \\
\hline$E_{l a b}[\mathrm{MeV}]$ & $\sigma_{e l}^{1}\left[\mathrm{fm}^{2}\right]$ & $\sigma_{e l}^{1+2}\left[\mathrm{fm}^{2}\right]$ & $\sigma_{e l}^{1+2+3}\left[\mathrm{fm}^{2}\right]$ & $\sigma_{e l}^{\mathrm{ND}}\left[\mathrm{fm}^{2}\right]$ & $\sigma_{e l}^{1}\left[\mathrm{fm}^{2}\right]$ & $\sigma_{e l}^{1+2}\left[\mathrm{fm}^{2}\right]$ & $\sigma_{e l}^{1}\left[\mathrm{fm}^{2}\right] \sigma_{e l}^{1+2}\left[\mathrm{fm}^{2}\right]$ \\
\hline \hline 100 & 40.66 & 44.47 & 30.27 & 26.53 & 29.58 & 28.24 & 32.60 & 30.83 \\
200 & 16.81 & 18.12 & 15.84 & 15.31 & 15.32 & 15.06 & 15.74 & 15.46 \\
500 & 6.87 & 7.04 & 6.82 & 6.74 & 6.59 & 6.52 & 6.63 & 6.56 \\
800 & 4.29 & 4.33 & 4.34 & 4.32 & 4.23 & 4.19 & 4.25 & 4.21 \\
1000 & 3.43 & 3.46 & 3.47 & 3.46 & 3.44 & 3.41 & 3.45 & 3.43 \\
1200 & 2.91 & 2.92 & 2.95 & 2.96 & 2.90 & 2.88 & 2.91 & 2.89 \\
1500 & 2.32 & 2.33 & 2.41 & 2.41 & 2.35 & 2.33 & 2.35 & 2.34 \\
2000 & 1.68 & 1.68 & 1.76 & 1.76 & 1.78 & 1.77 & 1.78 & 1.77 \\
\hline \hline
\end{tabular}

TABLE II: The Total cross section for elastic scattering for the first 3 terms of the Faddeev multiple scattering series, the fully converged Faddeev calculation, and the first two orders of Glauber calculations based on choices (a) and (b) for the unit vector $\hat{n}$ The superscripts indicate the order in the two-body $t$ matrix.

\begin{tabular}{|c|ccc||ccc|}
\hline & \multicolumn{3}{|c|}{ Faddeev } & \multicolumn{3}{c|}{ Glauber } \\
\hline$E_{\text {lab }}[\mathrm{MeV}]$ & $\sigma_{\text {tot }}^{\mathrm{ND}}\left[\mathrm{fm}^{2}\right]$ & $\sigma_{e l}^{\mathrm{ND}}\left[\mathrm{fm}^{2}\right]$ & $\sigma_{b r}^{\mathrm{ND}}\left[\mathrm{fm}^{2}\right]$ & $\sigma_{\text {tot }}^{\mathrm{Gl}}\left[\mathrm{fm}^{2}\right]$ & $\sigma_{b r}^{\mathrm{Gl}}(\mathrm{a})\left[\mathrm{fm}^{2}\right] \sigma_{b r}^{\mathrm{Gl}}(\mathrm{b})\left[\mathrm{fm}^{2}\right]$ \\
\hline \hline 100 & 34.16 & 26.53 & 7.63 & 29.00 & 0.76 & - \\
200 & 19.00 & 15.31 & 3.69 & 17.39 & 2.33 & 1.93 \\
500 & 10.30 & 6.74 & 3.56 & 9.49 & 2.97 & 2.93 \\
800 & 7.22 & 4.32 & 2.90 & 6.77 & 2.58 & 2.56 \\
1000 & 6.00 & 3.46 & 2.54 & 5.74 & 2.33 & 2.31 \\
1200 & 5.24 & 2.96 & 2.27 & 5.01 & 2.13 & 2.12 \\
1500 & 4.37 & 2.41 & 1.97 & 4.22 & 1.89 & 1.88 \\
2000 & 3.35 & 1.76 & 1.59 & 3.36 & 1.56 & 1.59 \\
\hline \hline
\end{tabular}

TABLE III: The total cross section and the total cross section for elastic scattering and breakup reactions as obtained from the exact Faddeev calculations are listed in the left part of the table. On the right side the total cross section as obtained from the Glauber calculation (including the second order correction) is given. The total cross sections for breakup reactions, $\sigma_{b r}$, is obtained by subtracting the total cross sections ((a) and (b)) for elastic scattering taken from Table 1 from the total cross section $\sigma_{\text {tot }}^{\mathrm{Gl}}$. 


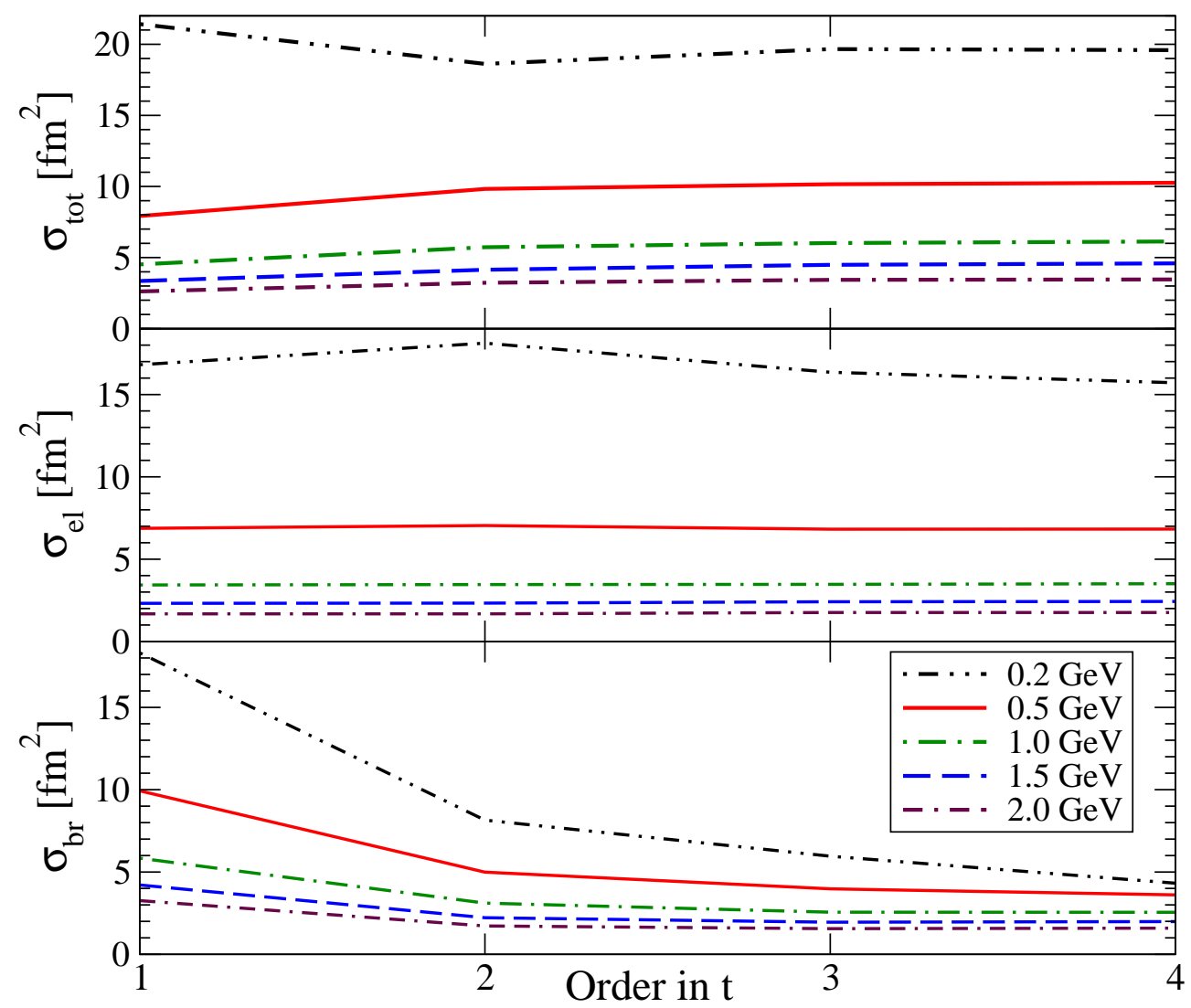

FIG. 1: (Color online) The total cross section (top panel), the elastic total (middle panel) and the breakup total cross section (bottom panel) as function of the order $t$ in the multiple scattering series for selected laboratory projectile energies indicated in the figure. Starting from the first order in the Faddeev calculation, the next three higher orders (rescattering terms) are successively added. 


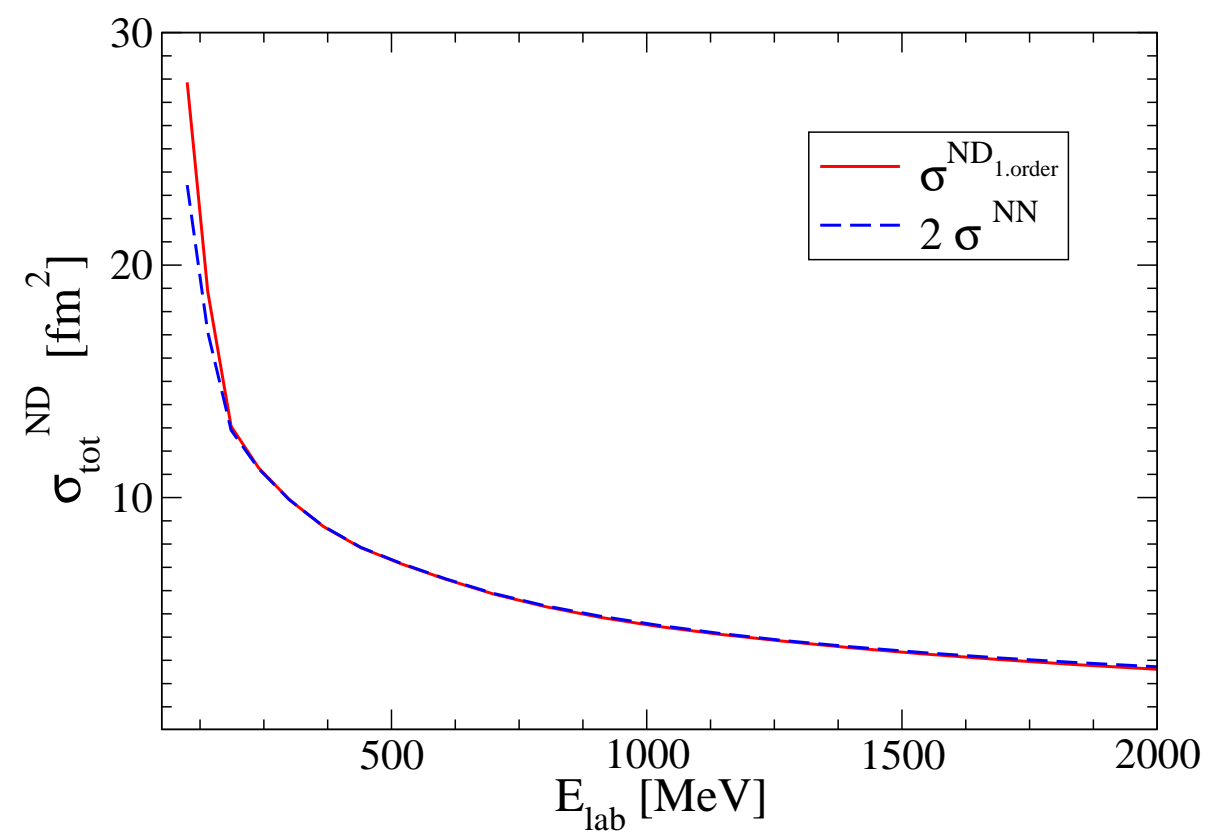

FIG. 2: (Color online) The total cross section for three-body scattering as function of the laboratory projectile energy. Shown are the first order (in $t$ ) Faddeev calculation and twice the two-body total cross section $\sigma^{\text {NN }}$ corresponding to the first order term in a Glauber calculation as well as in the expansion of Ref. [24].

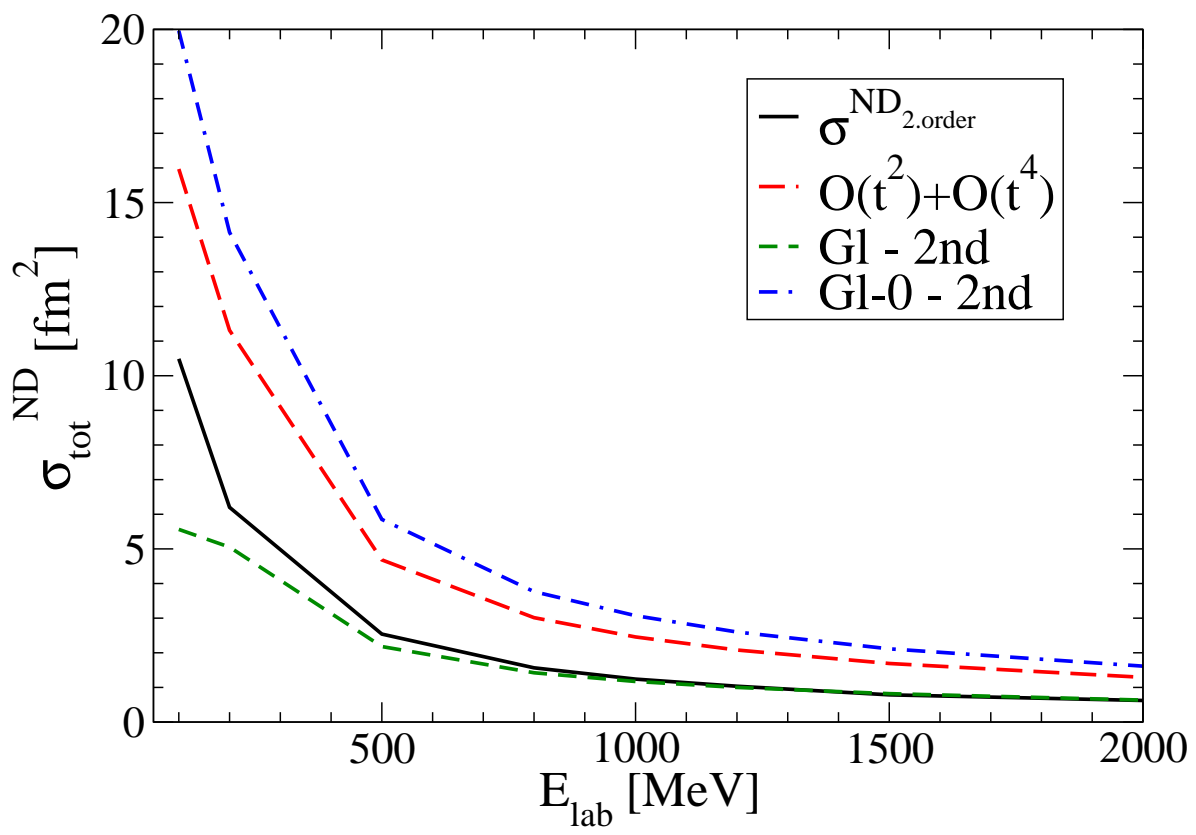

FIG. 3: (Color online) The second order correction to the total cross section for three-body scattering as function of the laboratory projectile energy. The solid line represents the second order term, $t P G_{0} t P$, of the Faddeev multiple scattering series and the long dashed line the correction obtained in Ref. 24 from the high energy limit of the $2^{\text {nd }}$ order Faddeev term. The short dashed line corresponds to the $2^{\text {nd }}$ order Glauber correction $\delta \sigma_{\text {tot }}$ from Eq. (3.32), while the dash-dotted line stands for the approximation of Eq. (3.33). 


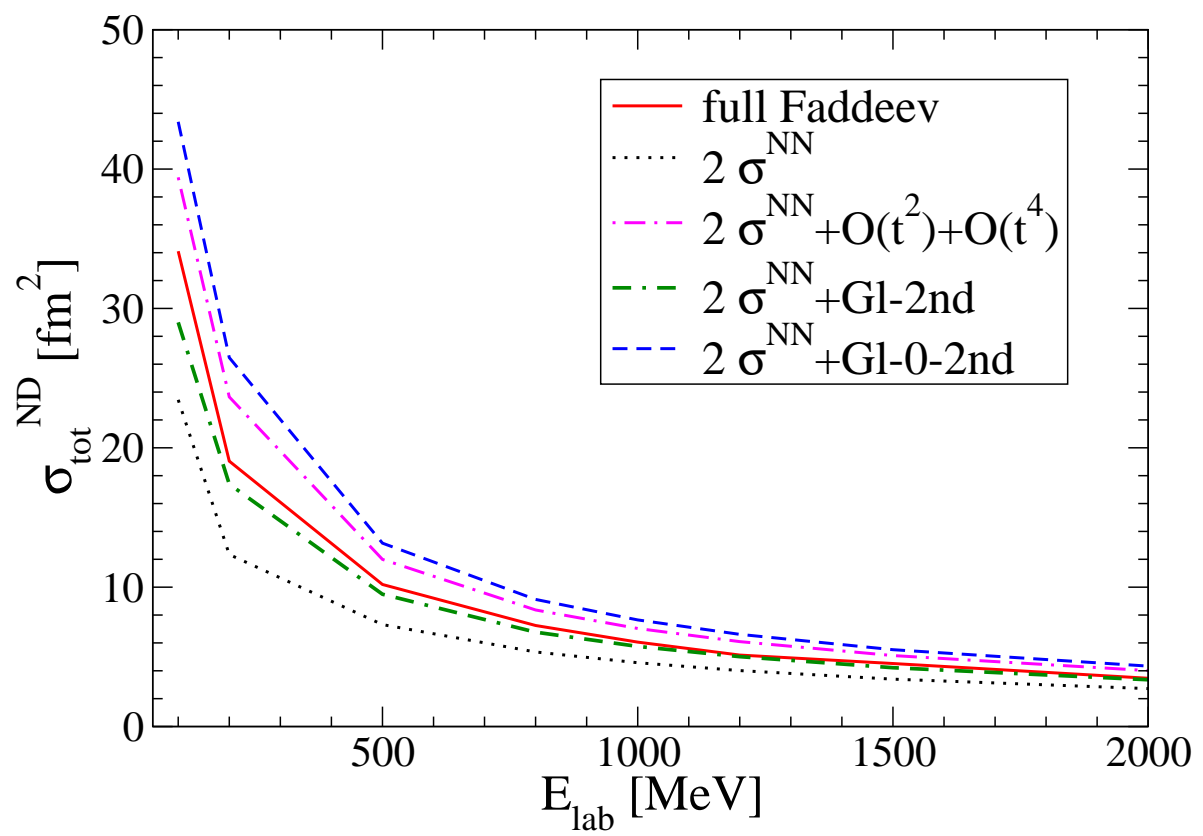

FIG. 4: (Color online) The total cross section for three-body scattering as function of the laboratory projectile energy. The solid line represents fully converged Faddeev calculations, the dotted line twice the two-body total cross section. To this are added the correction as extracted from the high energy limit of the $2^{\text {nd }}$ order Faddeev term from Ref. 24] (dash-dotted line), the second order Glauber correction $\delta \sigma_{\text {tot }}$ (dash-dash-dotted line) and its approximation (dashed line).

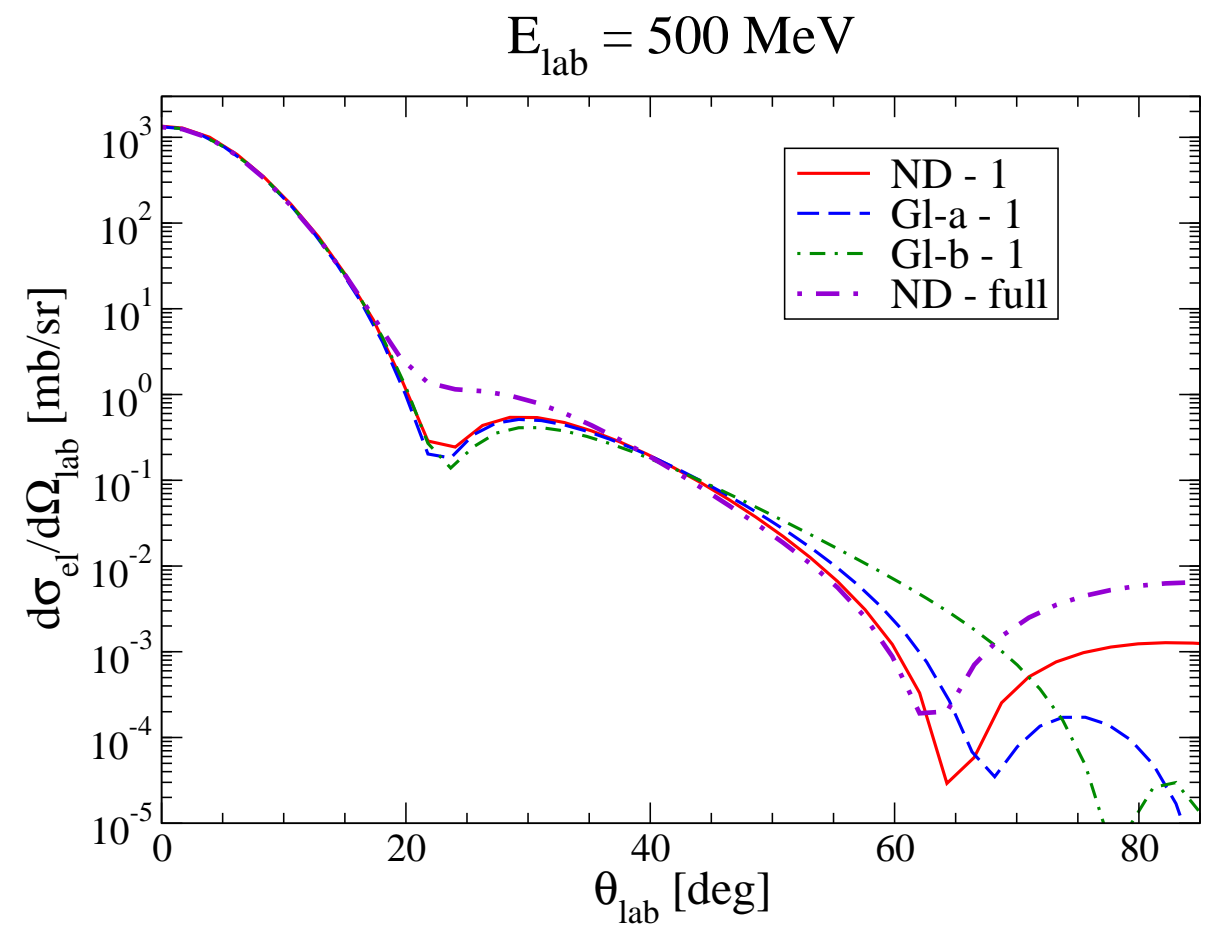

FIG. 5: (Color online) The differential cross section for elastic three-body scattering at 500 MeV laboratory projectile energy as function of the laboratory scattering angle. The solid line represent the first order Faddeev calculation. The dashed and dash-dotted line stand for the first order Glauber calculations for the two choices (a) and (b) of the direction of the unit vector $\hat{n}$ (see text). For comparison, the dash-double-dotted line represents the full Faddeev calculation. 


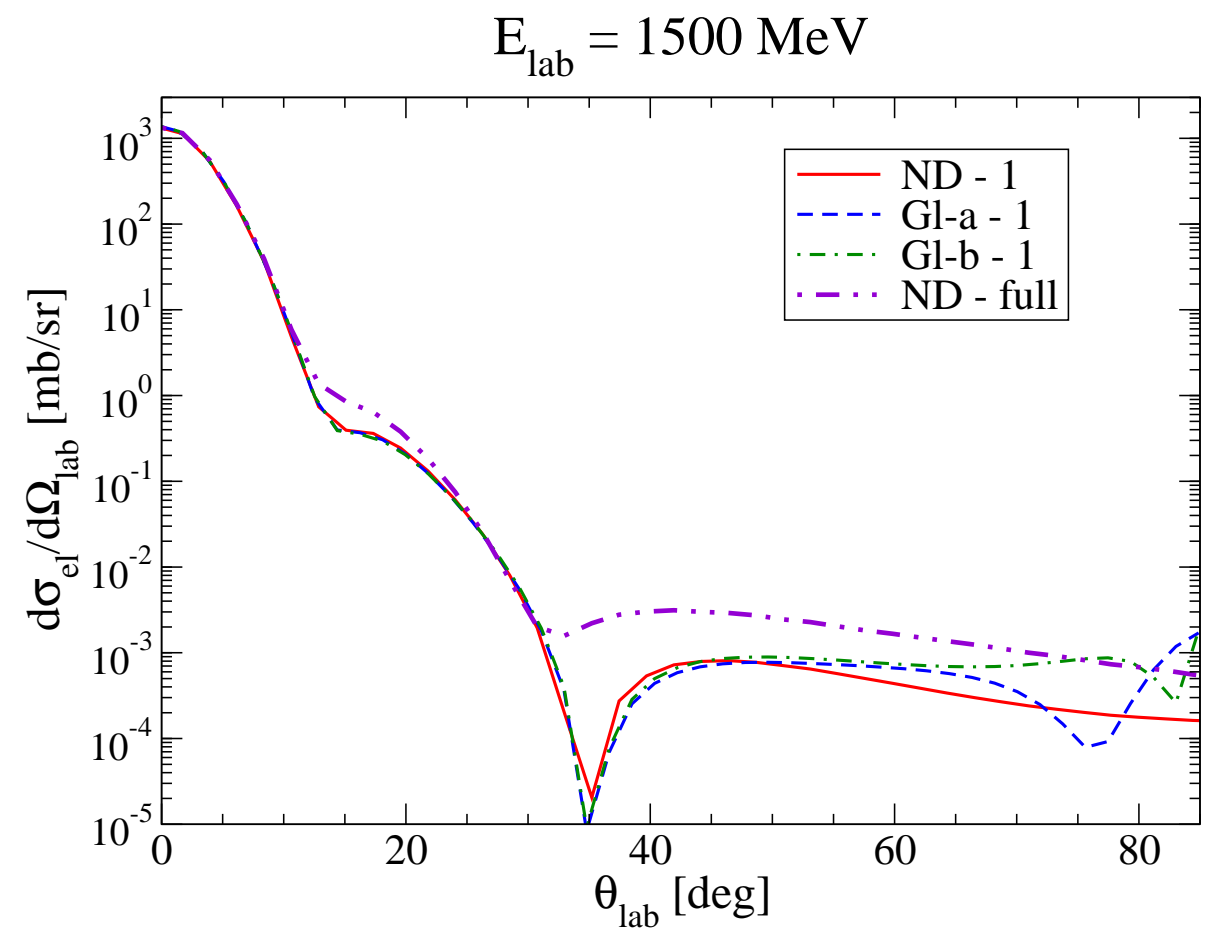

FIG. 6: (Color online) The differential cross section for elastic three-body scattering at 1500 MeV laboratory projectile energy as function of the laboratory scattering angle. The meaning of the curves is the same as in Fig. 5.

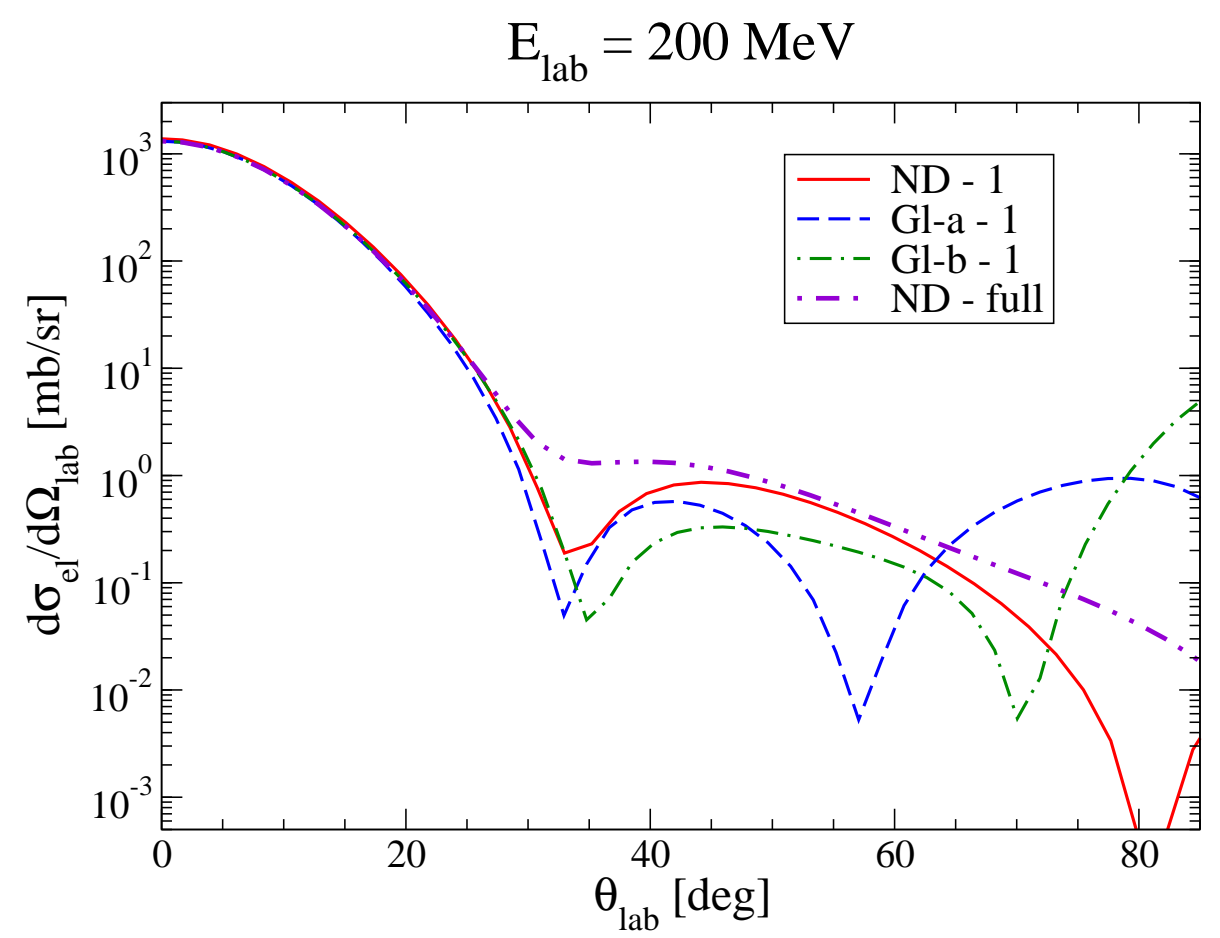

FIG. 7: (Color online) The differential cross section for elastic three-body scattering at $200 \mathrm{MeV}$ laboratory projectile energy as function of the laboratory scattering angle. The meaning of the curves is the same as in Fig. 5. 


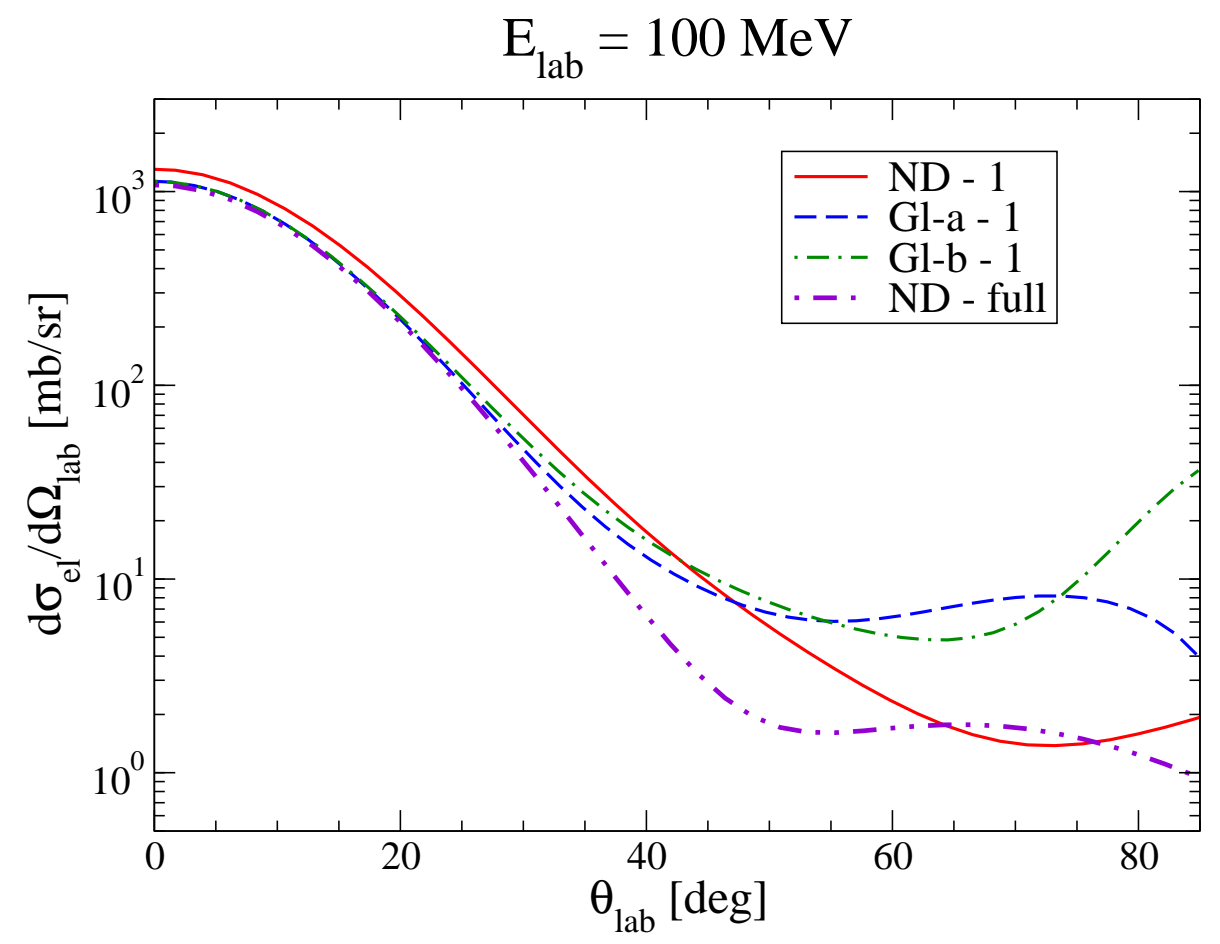

FIG. 8: (Color online) The differential cross section for elastic three-body scattering at 100 MeV laboratory projectile energy as function of the laboratory scattering angle. The meaning of the curves is the same as in Fig. 5.

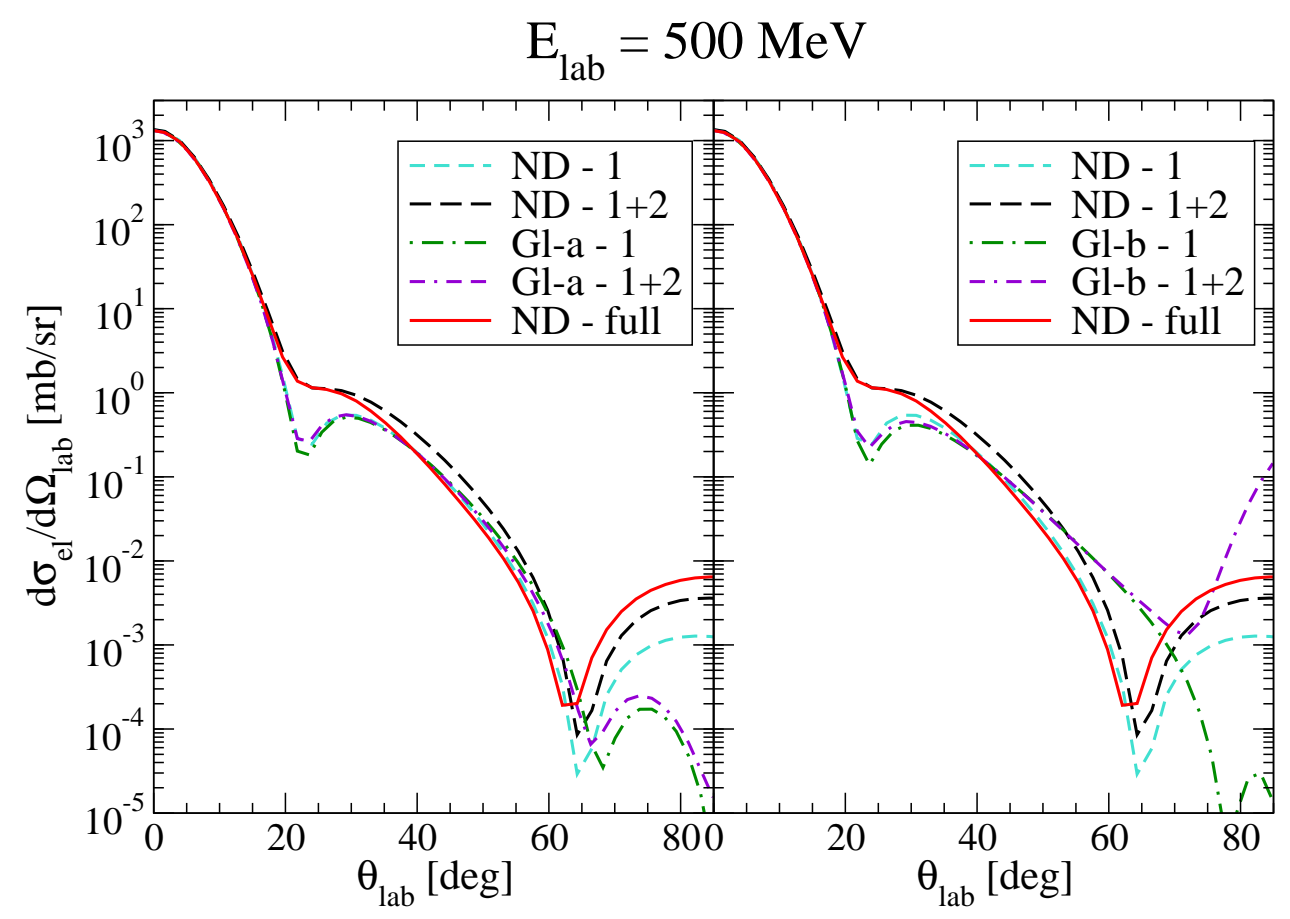

FIG. 9: (Color online) The differential cross section for elastic three-body scattering at 500 MeV laboratory projectile energy as function of the laboratory scattering angle. In both panels short dashed line represents the first order Faddeev calculation, the long dashed line the first and second order are considered, and the solid line stands for the full Faddeev calculation. The left panel shows the first order (dash-dotted line) and first plus second order (double-dash-dotted line) Glauber calculation for the choice (a) of the direction of the unit vector $\hat{n}$ (see text). The right panel contains the same for the choice (b). 
$\mathrm{E}_{\text {lab }}=1000 \mathrm{MeV}$

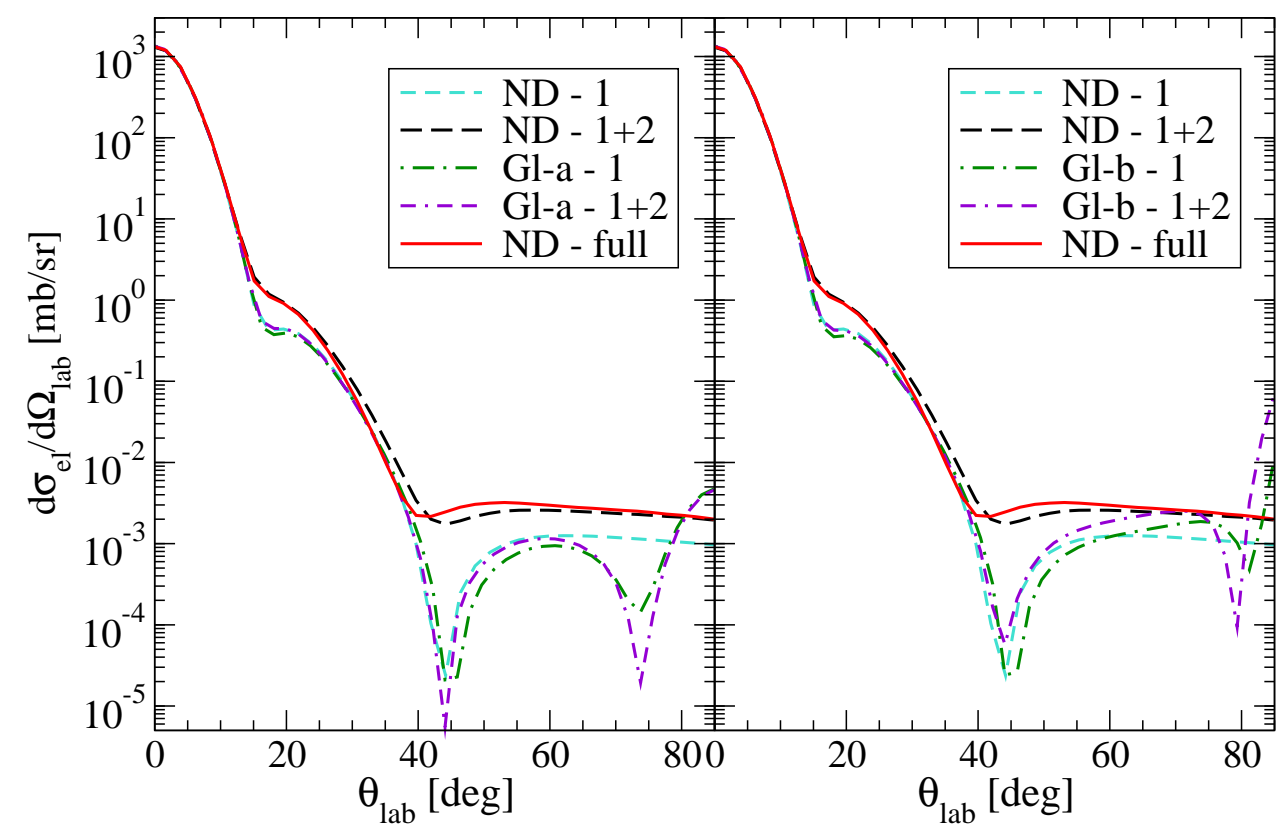

FIG. 10: (Color online) The differential cross section for elastic three-body scattering at 1000 MeV laboratory projectile energy as function of the laboratory scattering angle. The curves have the same meaning as in Fig. 9.

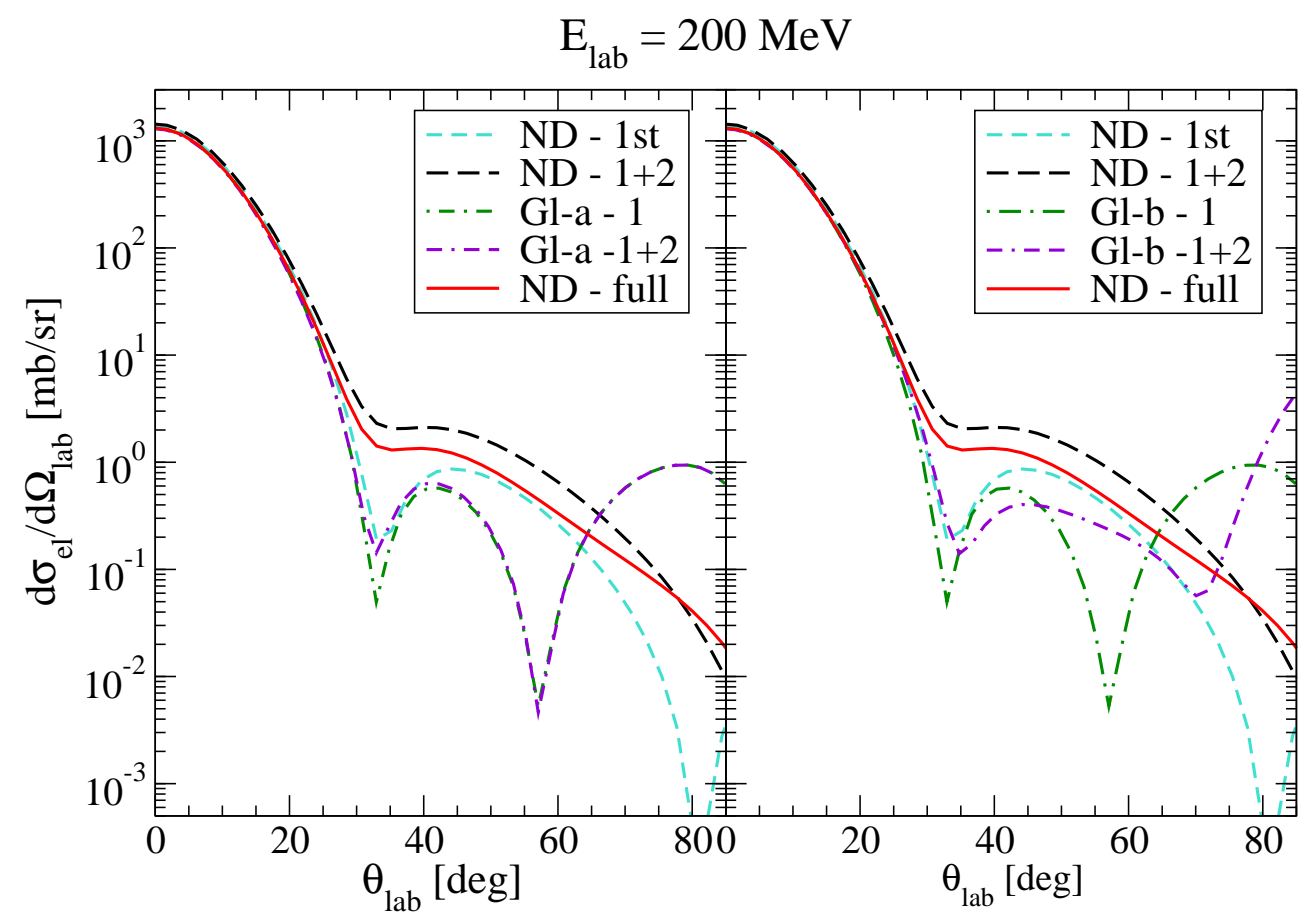

FIG. 11: (Color online) The differential cross section for elastic three-body scattering at 200 MeV laboratory projectile energy as function of the laboratory scattering angle. The curves have the same meaning as in Fig. 9. 


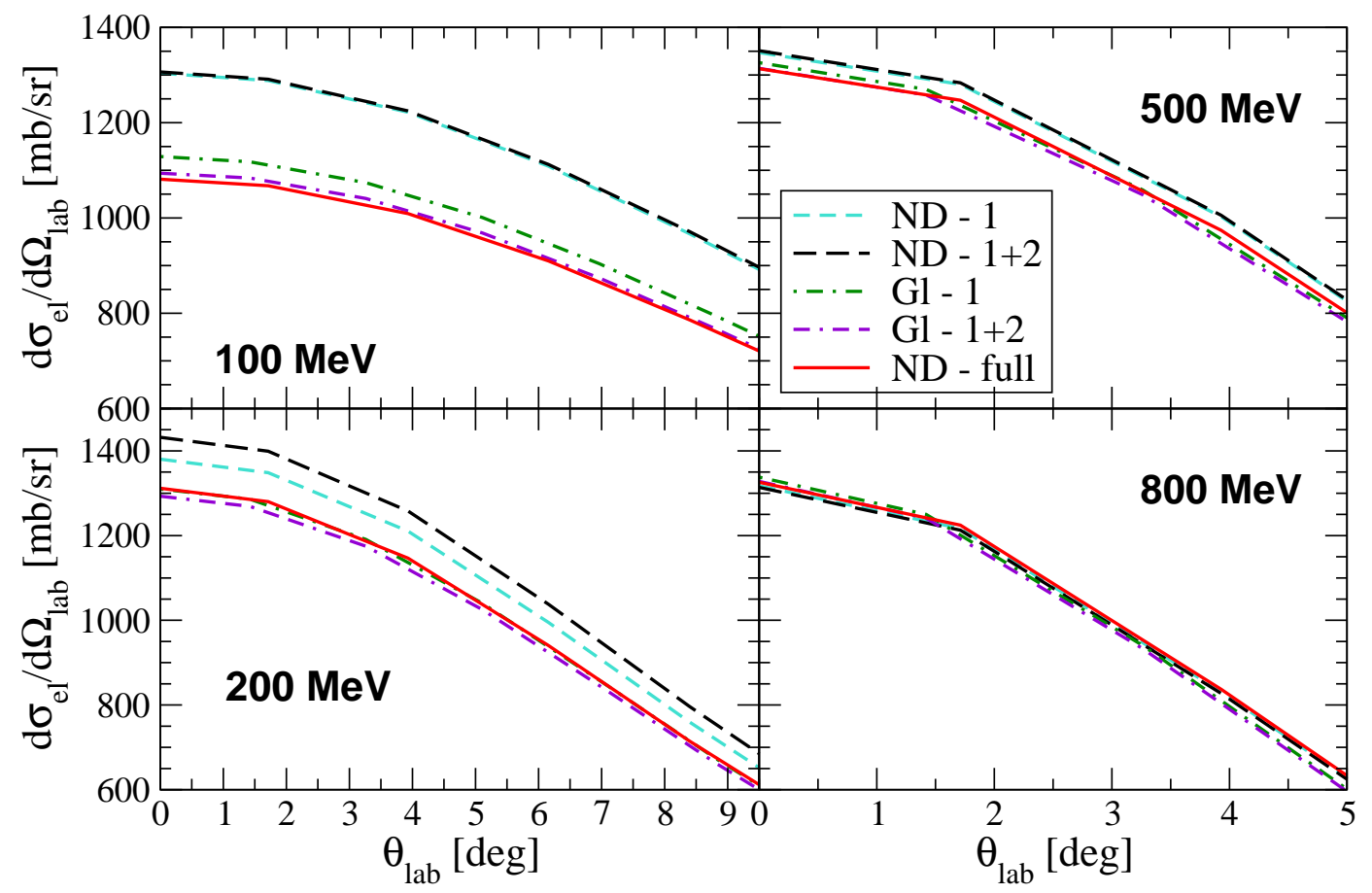

FIG. 12: (Color online) The differential cross section for elastic three-body scattering in forward direction for $100 \mathrm{MeV}$ (left upper panel), $200 \mathrm{MeV}$ (left lower panel), $500 \mathrm{MeV}$ (right upper panel), and $800 \mathrm{MeV}$ (right lower panel) laboratory projectile energy. The short dashed line represents the first order Faddeev calculation, the long dashed line the first and second order are considered, and the solid line stands for the full Faddeev calculation. The first order and first plus second order Glauber calculation for the choice (b) of the direction of the unit vector $\hat{n}$ are given by the dash-dotted and double-dash-dotted lines. 